\title{
Experimental study of rf pulsed heating
}

\author{
Lisa Laurent, ${ }^{*}$ Sami Tantawi, Valery Dolgashev, and Christopher Nantista \\ SLAC National Accelerator Laboratory, 2575 Sand Hill Road, Menlo Park, California 94025, USA \\ Yasuo Higashi \\ KEK, High Energy Accelerator Research Organization, 1-1 Oho, Tsukuba, Ibaraki 305-0801, Japan
}

Markus Aicheler, Samuli Heikkinen, and Walter Wuensch

CERN, European Organization for Nuclear Research, 1211 Geneva 23, Switzerland

(Received 9 September 2010; published 7 April 2011)

\begin{abstract}
Cyclic thermal stresses produced by rf pulsed heating can be the limiting factor on the attainable reliable gradients for room temperature linear accelerators. This is especially true for structures that have complicated features for wakefield damping. These limits could be pushed higher by using special types of copper, copper alloys, or other conducting metals in constructing partial or complete accelerator structures. Here we present an experimental study aimed at determining the potential of these materials for tolerating cyclic thermal fatigue due to rf magnetic fields. A special cavity that has no electric field on the surface was employed in these studies. The cavity shape concentrates the magnetic field on one flat surface where the test material is placed. The materials tested in this study have included oxygen free electronic grade copper, copper zirconium, copper chromium, hot isostatically pressed copper, single crystal copper, electroplated copper, Glidcop ${ }^{\circledR}$, copper silver, and silver plated copper. The samples were exposed to different machining and heat treatment processes prior to rf processing. Each sample was tested to a peak pulsed heating temperature of approximately $110^{\circ} \mathrm{C}$ and remained at this temperature for approximately $10 \times 10^{6} \mathrm{rf}$ pulses. In general, the results showed the possibility of pushing the gradient limits due to pulsed heating fatigue by the use of copper zirconium and copper chromium alloys.
\end{abstract}

DOI: 10.1103/PhysRevSTAB.14.041001

PACS numbers: 29.20.Ej, 52.80.Pi, 52.80.Vp

\section{INTRODUCTION}

Cyclic thermal stresses produced by rf pulsed heating was originally considered a limiting factor for linear accelerators at extremely high frequencies [1,2]. This motivated experimental studies of the phenomenon [3-6]. However, pulsed heating limited the performance of accelerator couplers at $\mathrm{x}$-band linear accelerators considered for the so-called next linear collider (NLC) and it was also credited for the degradation of $\mathrm{x}$-band klystron output cavities. Studies of accelerator structures for future linear colliders demanded strong damping of wakefields caused by higher order modes [7]. Adding damping features to these structures typically enhances surface rf magnetic fields or currents and, hence, thermal stresses. In these structures the thermal stresses could potentially limit the reliable operating gradient. Furthermore, recent studies of breakdown rates in high gradient linear accelerators showed a direct correlation between these rates and pulsed

\footnotetext{
*laurent@slac.stanford.edu
}

Published by American Physical Society under the terms of the Creative Commons Attribution 3.0 License. Further distribution of this work must maintain attribution to the author(s) and the published article's title, journal citation, and DOI. heating [8]. Geometrical variations of accelerator structures aimed at reducing surface rf magnetic fields can only go so far. The only alternative after that is to totally or partially change the structure material. This is the original motivation of this study. This work differs from that published in Ref. [3] in two aspects. First, we study materials other than oxygen-free high conductivity (OFHC) copper. Second, our experimental setup utilizes a special cavity that permits the testing of the materials without damage to the cavity itself, which is made of OFHC copper. This is done by a choice of geometry that has no electric fields on the walls and has substantially reduced magnetic fields on all surfaces except that of the material under test. Finally, we couple to the cavity with a specially designed mode converter [9] which generates the $\mathrm{TE}_{01}$ mode in a circular guide and, hence, eliminates any surface electric field at the coupling port. This made the cavity extremely reliable and the data is only related to the pulsed heating affects and nothing else. This work has shown that pulsed heating surface damage in vacuum is dependent on processing time, pulsed heating temperature, material hardness, and crystallographic orientation. At low temperatures, grain boundaries were the initial sites impacted by pulsed heating and at higher temperatures the surface damage (e.g. fatigue cracking) due to cyclic thermal stresses varied 
depending on the material tested. In general, the level of pulsed heating surface damage was less on harder materials which usually have smaller grains.

\section{EXPERIMENTAL SETUP}

\section{A. Cavity design}

To test materials for pulsed heating effects only, it is desirable to eliminate any experimental limitations that might be imposed by surface electric field. To avoid potential problems caused by rf breakdown, multipacting, and heating due to dark current, a test cavity should utilize a $\mathrm{TE}_{0 n m}$-type mode in an axially symmetric geometry. The electric field lines in such modes form self-closing axial rings which do not terminate on any surface. A further benefit of these modes arises from the fact that their wall currents are also purely azimuthal, making them insensitive to small longitudinal gaps. This facilitates the incorporation of a test sample end plate, which need not have good electrical contact with, or even touch, the main body of the cavity.

\section{Mushroom cavity}

The approach we took is to focus the magnetic field solely on the bottom plate, formed by the test sample. The geometry arrived at after some simulation effort is shown in Fig. 1, along with electric and magnetic field patterns. The mode utilized is $\mathrm{TE}_{013}$-like, but the upper portion of the cavity flares out in a sort of mushroom shape, allowing the upper lobes of the mode to spread out over greater surface area. The cavity is approximately $4.88 \mathrm{~cm}$ high,
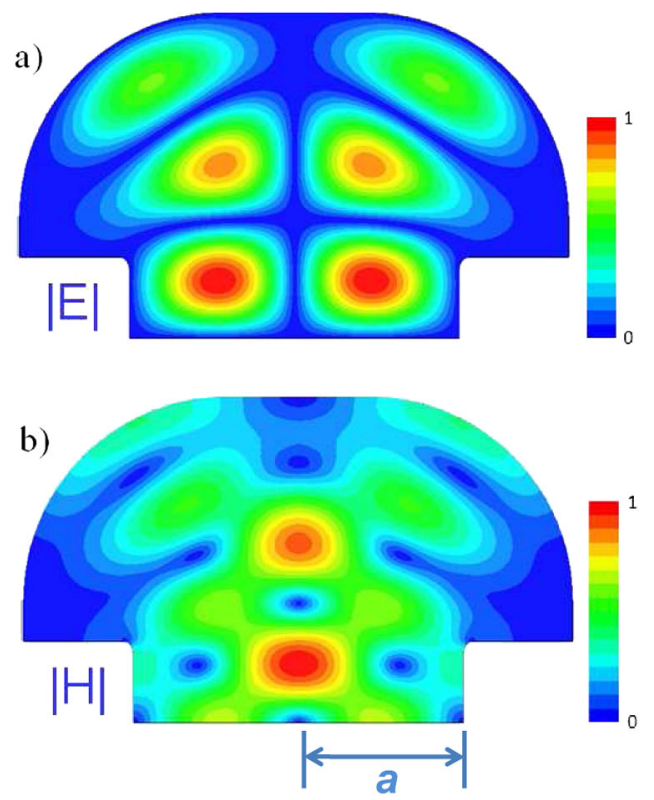

FIG. 1. Geometry of the pulsed heating test cavity and normalized color plots of the (a) E-field and (b) H-field patterns for the $\mathrm{TE}_{013}$-like mode. The bottom edge is the face of the test sample.

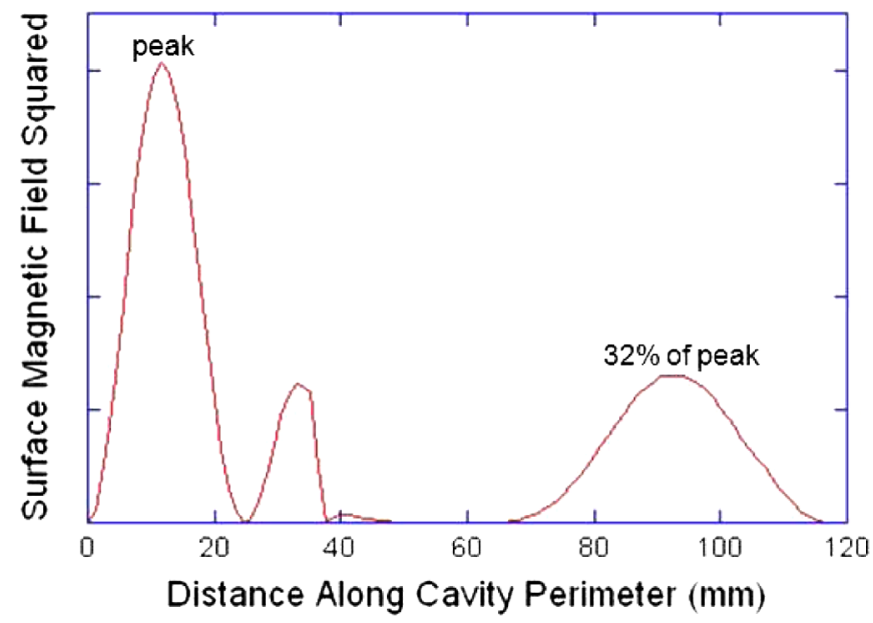

FIG. 2. Magnetic field profile (in arbitrary units) along the contour of the cavity wall, beginning at the center of the bottom face. The first peak, and by far the highest, is on the bottom face.

with a maximum diameter of $8.255 \mathrm{~cm}$. The cavity bottom, to be formed by connecting a flange with the sample attached, has a diameter of only $4.98 \mathrm{~cm}$.

The square of the magnetic field profile along the cavity wall, proportional for a given conductivity to pulsed heating, is plotted in Fig. 2, starting at the axis on the bottom and going out and around to the top. The field reaches a peak on the bottom plate significantly higher than anywhere else. With the next highest surface fields, on the lower side and the curved area near the top, reaching only $56 \%$ of this, the off-sample pulsed heating is suppressed by better than a factor of 3 . As noted, the surface electric field is everywhere zero.

In a later redesigned version of this cavity, the next highest surface magnetic field was further reduced to $41 \%$ of the peak, almost another factor of 2 in pulsed heating. This was done by fully extending the curvature of the dome while reducing the mushroom stem height to a minimal thickness $(2.54 \mathrm{~mm}$ ) for an iris to cover the sample edge. The result was nearly a perfect hemisphere, supporting a three-lobed mode with the central lobe on the slightly recessed sample. In fact, we realized that the optimal cavity shape should have been a perfect hemisphere which we are currently building for the next series of experiments.

\section{Coupling}

The cavity includes only one rf coupling, through a centered circular aperture in the top. A second coupling aperture would be useful for diagnostics, but would need to either be made through the center of the material sample or break the axial symmetry of the cavity. The former would unduly complicate the preparation of samples and introduce on them a machined edge on which the field would be locally enhanced, adding uncertainty to the peak value. The ability to test materials with a simple flat, replaceable, 
three-inch disk (with screw holes beyond the cavity region), is a great asset of this test setup. The latter coupling option of breaking symmetry by coming in through the side in an overmoded, high- $Q$ resonator will introduce uncontrolled higher order modes. This was used in [3] and resulted in complicated coupling problems and rf breakdown on irises. Also, the broken symmetry necessitated the use of spring contacts near the material sample-cavity interface, which also caused problems during high-power operations. Unintentional excitation of nonaxisymmetric cavity modes and evanescent fields from such an aperture or probe could affect the $Q$ and alter the surface fields. This would limit the accuracy and usefulness of the experimental results. Thus, the cavity's simplicity is maintained by using only one waveguide penetration.

As described below, we are able with one port to determine the quality factor well enough from $S_{11}$, using low-power frequency data along with high-power measurements, although this method is not as straightforward as measuring $S_{21}$ resonance widths. The flat region maintained on top of the cavity dome facilitated modeling and machining of an iris of the desired coupling through a wall of constant thickness $(\sim 1 / 8 \mathrm{~cm})$. The electric and magnetic fields are both zero at the top center of the cavity. Furthermore, the flared shape of the cavity pulls the upper lobe of the mode away from the center, requiring a rather large hole, nearly a centimeter in radius, to achieve critical coupling. This corresponds to a loaded quality factor of 22800 at room temperature. The dependence of the external $Q$ on iris radius roughly followed a 13th power dependence over 8 orders of magnitude, according to HFSS [10] calculations. This sensitivity makes our geometry ideal for approaching critical coupling with a reasonable hole radius over a wide range of unloaded $Q$ 's. It also tends to overcouple other modes.

To couple to this cavity mode through a centered iris requires the same field pattern on the waveguide side, namely an incident $\mathrm{TE}_{01}$ wave. Furthermore, to avoid excitation of other potentially high- $Q$ cavity modes, this waveguide mode should be as pure as possible. We used a mode converter of a new design to [9] launch $\mathrm{TE}_{01}$ in a 1.5 -inch diameter waveguide, connected by a vacuum flange to the top of the cavity. The device has been designed for and tested at extremely high-power levels, several hundreds of megawatts at x-band [11]. The input of the mode converter is standard WR90 rectangular waveguide. A very compact section converts the fundamental $\mathrm{TE}_{10}$ mode to $\mathrm{TE}_{20}$. This is followed by a height taper and a multisection taper from rectangular to circular cross-section designed to launch high-purity $\mathrm{TE}_{01}$. Simulation shows resulting parasitic mode relative levels to be on the order of $-60 \mathrm{~dB}$.

\section{Low-power cavity characterization}

Most techniques used to interpret the reflection data from a high quality factor resonator are based on fitting the so-called $Q$ circle (see [12] and the references cited therein for a review of these techniques). Briefly, the cavity frequency, $f_{0}$ is calculated from the minimum of the cavity frequency response. Then, the coupling parameter, $\beta$, and the attenuation of the transfer waveguide, $e^{-\alpha L}$, where $\alpha$ is the waveguide attenuation constant and $L$ is the length, are calculated by fitting the measured $S_{11}$ response to a cavity model that has the following frequency response:

$$
S_{11}(f)=e^{-2 L\left(i k_{g}+\alpha\right)}\left(\frac{2 i \beta}{(\beta+1)\left(f_{s} Q_{L}-i\right)}+1\right),
$$

where $k_{g}$ is $2 \pi$ over the guide wavelength and

$$
f_{s}=f / f_{0}-f_{0} / f
$$

which represents a circle in the complex $S_{11}$ domain. Finally, the loaded quality factor $Q_{L}$ is calculated by inverting Eq. (1) and fitting a constant for $Q_{L}$ over the range of $f_{s}$.

\section{High-power cavity characterization}

The high-power tests are performed using a SLAC XL4 $50 \mathrm{MW}$ x-band klystron operating at $11.42 \mathrm{GHz}$ [13]. Because there is no circulator available at this frequency and power level, we use a long stainless steel waveguide with an attenuation of about $4 \mathrm{~dB}$ to provide $8 \mathrm{~dB}$ round trip isolation between the klystron and the cavity. A pulse, very close in shape to a square pulse, is injected into the cavity. The reflection coefficient is then measured through a set of directional couplers.

To eliminate the influence of the finite directivity of the coupler, the cavity is characterized through its response after the incident pulse is over. Since the cavity has a loaded $Q$ of about 23000 , the power decay time constant is about $2 \mu \mathrm{s}$, which is easily observable. Both phase and amplitude of the cavity response during the decay are measured. Based on this measured phase slope, the driver frequency is adjusted to follow the cavity frequency. This feedback is automated through a desktop computer. The amplitude of the cavity response during the decay time is fitted with an exponential curve to determine the $Q_{L}$. These high-power measurements are compared for accuracy to the low-power $Q_{L}$ measurements obtained for each sample.

Because the $8 \mathrm{~dB}$ isolation between the cavity and the klystron is not sufficient, an additional feedback loop is added to help maintain stable amplitude and phase across the input pulse flat top. This system is similar to that used to regulate SLED-II as described in [11]. A schematic diagram of the system is shown in Fig. 3.

From the cavity parameters determined at low power, the field profile from the simulations and the measured amplitude of the high-power input pulse, one can calculate the pulsed temperature rise pattern across the flat plate made of the material under test. An additional feedback loop is added to guarantee a constant temperature profile 


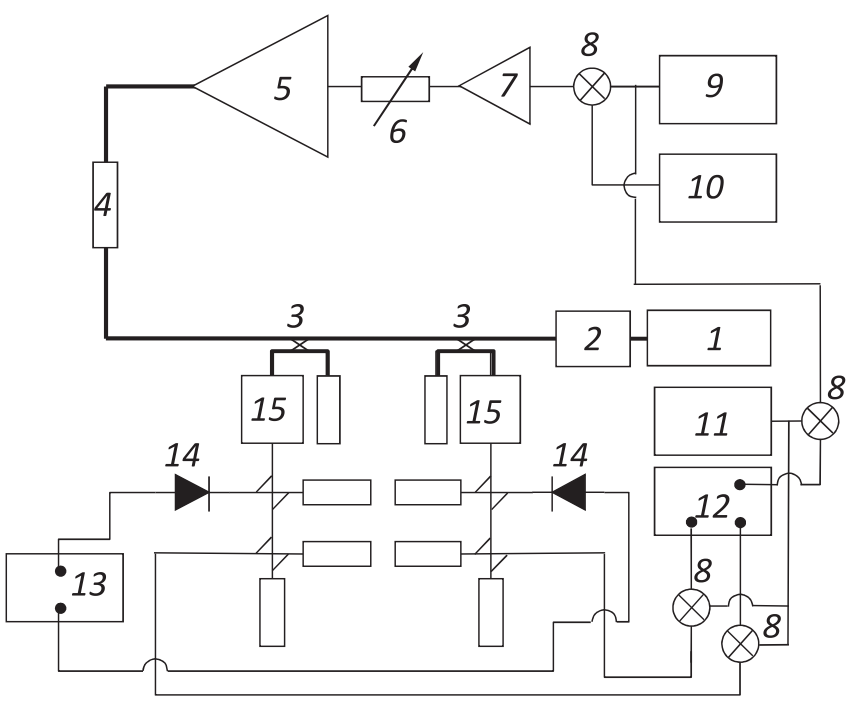

FIG. 3. Experimental setup schematic diagram: 1-pulse heating cavity, 2-mode converter creating $\mathrm{TE}_{01}$ mode, 3-highpower directional couplers, 4-high-power attenuator, 5-SLAC 50 MW klystron, 6-variable waveguide attenuator, $7-1.5 \mathrm{~kW}$ traveling wave tube amplifier, 8-mixer, 9-11.4 GHz synthesized signal generator, 10-arbitrary function generator, 11-local oscillator to drive mixers, 12-digital oscilloscope, 13-peak power meter, 14-power meter probes, 15-low pass filters. Not numbered: crossguide directional couplers that couple signal between low pass filter (15) and probes (14) and low-power rf loads which are connected to high-power directional couplers (4), and crossguide directional couplers.

on the sample. Figure 4 shows the mechanical design of the cavity and a picture of the end plate that is attached to the cavity using a high-power vacuum flange. The three-inch diameter pulsed heating sample is mounted onto the cavity end plate. A photograph of the cavity assembly is shown in

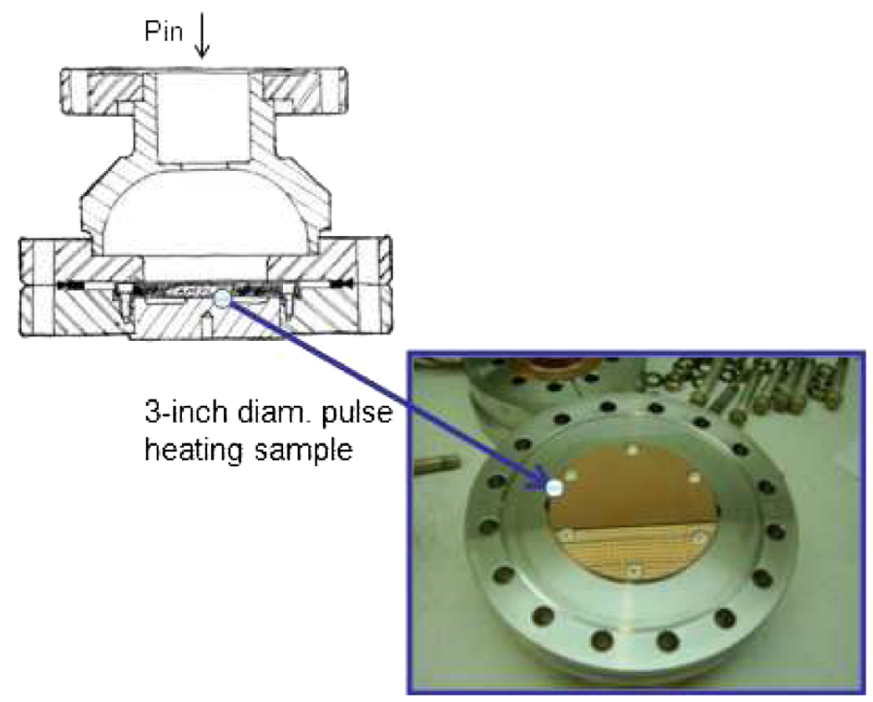

FIG. 4. The pulsed heating experiments are conducted using a $\mathrm{TE}_{01}$ cavity. The three-inch diameter samples are first mounted onto a cavity end cap (bottom right).

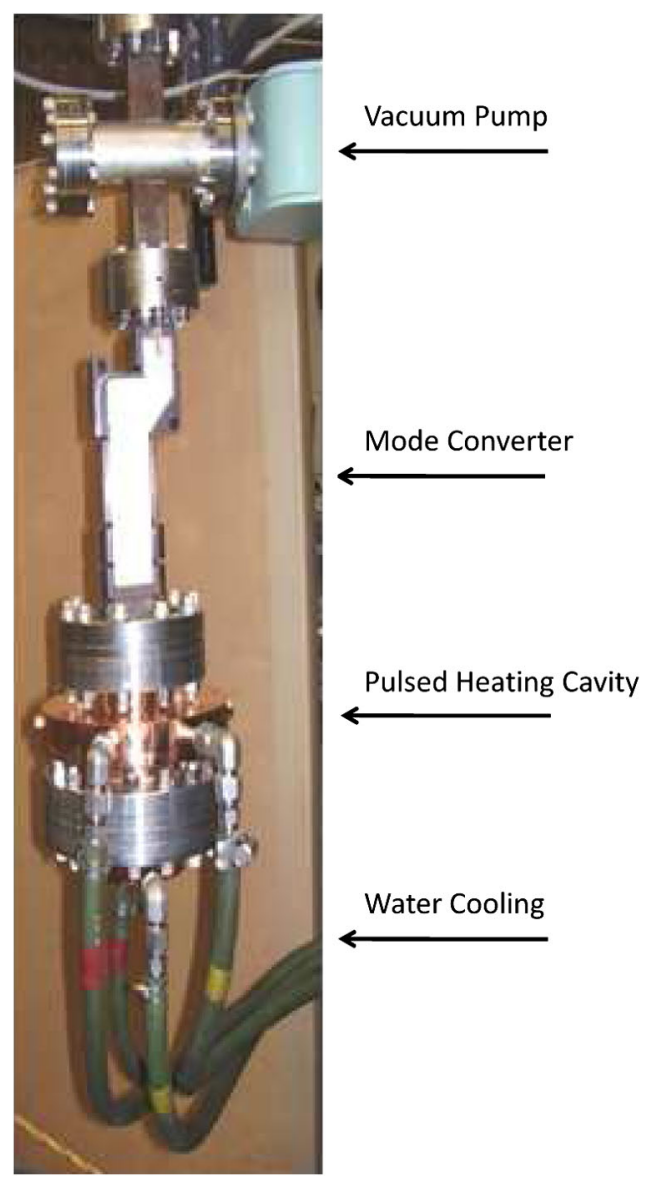

FIG. 5. The cavity has water cooling and is connected to the power source through a $\mathrm{TE}_{01}$ (WR90) to $\mathrm{TE}_{01}$ mode converter.

Fig. 5. The cavity is connected to the mode converter and to the cooling system which serves to hold the steady state cavity temperature constant.

The peak pulsed heating temperature on these test samples is not radially uniform in the $\mathrm{TE}_{01}$ pulsed heating ring. At each respective power level the peak temperature $\left(T_{\max }\right)$ is located at the center of the pulsed heating ring. The temperature distribution across the ring is proportional to $J_{1}^{2}\left(\frac{x}{a} r\right)$ where $J_{1}$ is the first order Bessel function of the first kind, $x=3.83171$, and $a$ is the diameter of the samples exposed to the rf. The maximum temperature $\left(T_{\max }\right)$ occurs at $r=0.481 a$. A normalized calculation of pulsed heating temperature as a function of radial distance is plotted in Fig. 6. The graph is superimposed on a photograph of a copper sample that was tested to a pulsed heating temperature of $110^{\circ} \mathrm{C}$. The pulsed heating ring on this sample is clearly visible in the high pulsed heating temperature region. The pulse length for these experiments is approximately $1.5 \mu \mathrm{s}$ at the input to the cavity with a pulse repetition rate of $60 \mathrm{~Hz}$.

An expression for the temperature rise as a function of time, $t$, and depth in the material, $z$, is derived in Ref. [14], Eq. 3.32. This expression can be simplified at the zero skin 


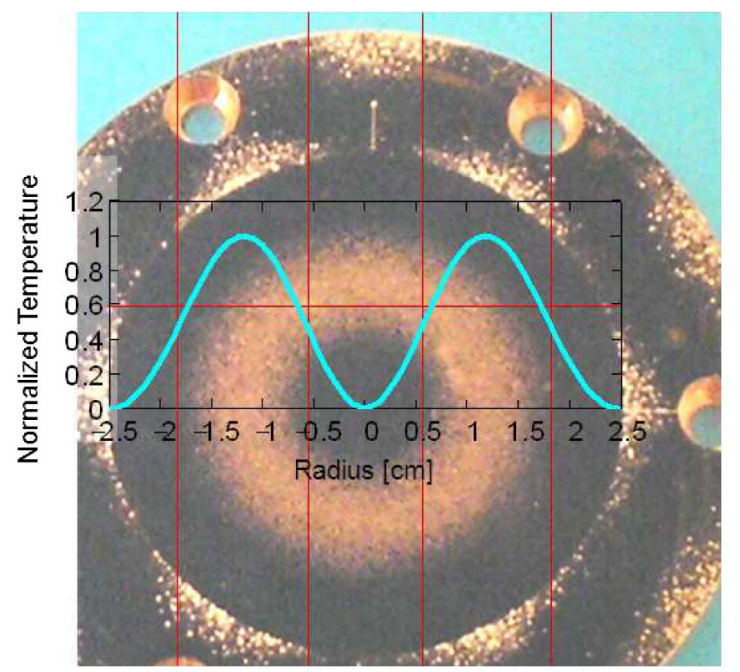

FIG. 6. Normalized pulsed heating temperature calculations superimposed on a copper pulsed heating sample.

depth limit, which is valid for small skin depths in comparison with the thermal diffusion length. In the samples we have tested, the skin depth is typically on the order of $0.65 \mu \mathrm{m}$, and the thermal diffusion length is greater than $10 \mu \mathrm{m}$. The simplified temperature rise function in this case is

$$
T(t, z)=\frac{R_{s}}{2\left(\sqrt{\pi \alpha} \rho c_{\epsilon}\right)} \int_{0}^{t} \frac{H^{2}\left(t^{\prime}, r\right) e^{-\left(z^{2} / 4 \alpha\left(t-t^{\prime}\right)\right)}}{\sqrt{t-t^{\prime}}} d t^{\prime}
$$

where $\alpha=\frac{k}{\rho c_{\epsilon}}$, the surface resistance $R s=\sqrt{\frac{\pi f \mu}{\sigma}}, k$ is the thermal conductivity, $\rho$ is material density, $c_{\epsilon}$ is specific heat at constant strain, $f$ is the frequency, $\mu$ is the magnetic permeability of the material under test, and $\sigma$ is the electrical conductivity. The surface magnetic field $H(t)$ as a function of time is given by:

$$
H(t, r)=H_{0}\left(\frac{J_{1}\left(x_{0} \frac{r}{a}\right)}{J_{1}\left(x_{1}\right)}\right)^{2} \begin{cases}1-e^{-(t / \tau)} & t \leq T_{p} \\ \left(e^{T_{p} / \tau}-1\right) e^{-(t / \tau)} & t>T_{p}\end{cases}
$$

where $x_{0}=3.83171, x_{1}=1.84118, \tau=\frac{2 Q_{\text {loaded }}}{\omega}$ is the filling time of the cavity, $T_{p}$ is the input pulse length, and $H_{0}$ is the maximum steady state magnetic field level in the cavity for a given input power. This value is calculated from the geometry of the cavity using the finite element code HFSS. A pulsed heating temperature of $110^{\circ} \mathrm{C}$ corresponds to $600 \mathrm{kA} / \mathrm{m}$. Ultrahigh gradient $\mathrm{x}$-band structures typically run at a pulsed heating temperature of $<50^{\circ} \mathrm{C}$ for low breakdown probability operation $\left(<10^{-2}\right.$ breakdown pulses/meter). For these accelerator structures (see Refs. $[15,16]$ ), the peak magnetic field is $<500 \mathrm{kA} / \mathrm{m}$ with a pulse length of $200 \mathrm{~ns}$. For further detailed discussions on the effect of pulsed heating on the breakdown rate in $\mathrm{x}$-band structures, the reader is referred to Refs. [15-18].

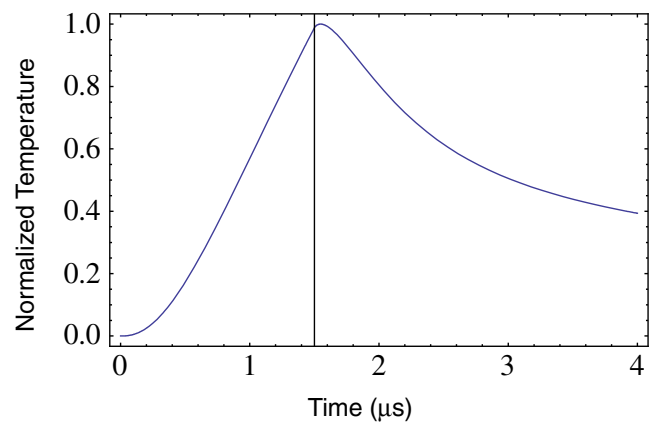

FIG. 7. Normalized temperature rise calculation on surface of test sample for a $1.5 \mu \mathrm{s}$ rf pulse as a function of time. The temperature is normalized to its maximum value at a given point on the surface.

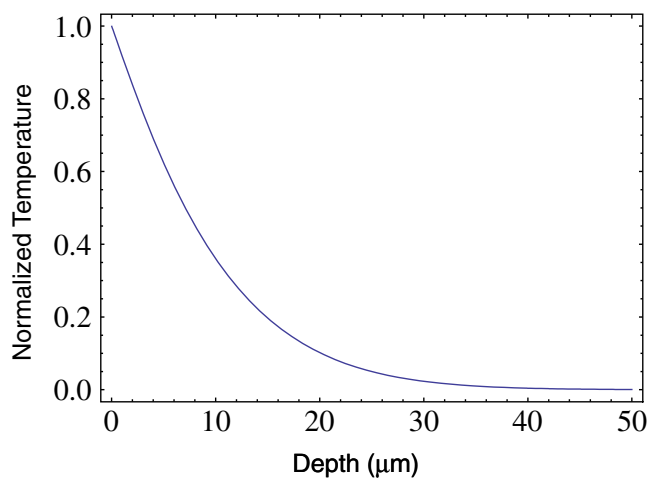

FIG. 8. Temperature profile at the maximum temperature point for a $1.5 \mu \mathrm{s}$ rf pulse as a function of surface depth.

The temperature expression, Eq. (3), is rather simple to evaluate numerically and was used to calculate the temperature rise for a given set of operational parameters. This was done on the fly during the testing process and the evaluated temperature was used on the feedback loop to adjust the power level of the klystron.

A temperature plot is shown in Fig. 7 for an rf pulse length of $1.5 \mu \mathrm{s}$. The temperature is normalized to its maximum value at a given point on the surface. The peak temperature inside the cavity occurs about $50 \mathrm{~ns}$ after the end of the rf pulse, shown by the vertical line on Fig. 7. The plot in Fig. 8 shows that the peak temperature approaches ambient temperatures at a depth of 30-40 $\mu \mathrm{s}$.

\section{PULSED HEATING RF TEST RESULTS}

\section{A. Initial experimentation to establish a testing protocol}

The first two pulsed heating samples tested were manufactured from C10100 oxygen-free electronic (OFE) grade, half hard, multiway forged copper. They were machined using diamond fly cutting and rinsed afterwards with alcohol. OFE copper is the metal generally used in the fabrication of room temperature high-power $\mathrm{rf}$ 


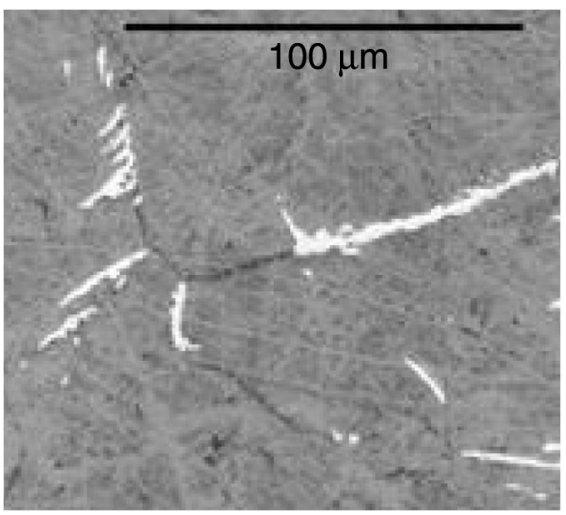

FIG. 9. Initial tests at a peak pulsed heating temperature of $70^{\circ} \mathrm{C}$ on OFE copper showed pulsed heating damage predominately followed grain boundaries.

components and accelerator structures and for this reason was the initial material tested to acquire baseline data. The first copper sample was initially processed to a peak pulsed heating temperature of $70^{\circ} \mathrm{C}$ for approximately $4 \times 10^{6} \mathrm{rf}$ pulses and then temporarily removed for surface analysis.

Pulsed heating surface damage was already evident at this temperature and was predominate along grain boundaries like that shown in the scanning electron microscope (SEM) image of Fig. 9. The same copper sample was reinstalled into the cavity and retested at the same pulsed heating temperature $\left(70^{\circ} \mathrm{C}\right)$ for an additional $4 \times 10^{6} \mathrm{rf}$ pulses. The $\mathrm{TE}_{01}$ pulsed heating ring was faintly visible after both of these runs and can be observed in the photographs of Fig. 10. An increase in the number of rf cycles during the 2nd run [Fig. 10(b)] showed many newly formed fatigue extrusions and propagation from fracture sites that were already initiated during the first run. Fatigue cracking results when a material is subjected to repeated cyclic stresses. Above a certain threshold, microscopic cracks will begin to form at the surface. SEM images showing a section of the pulsed heating ring after the first and second

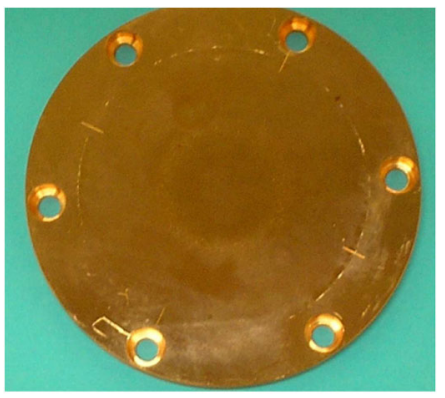

(a)

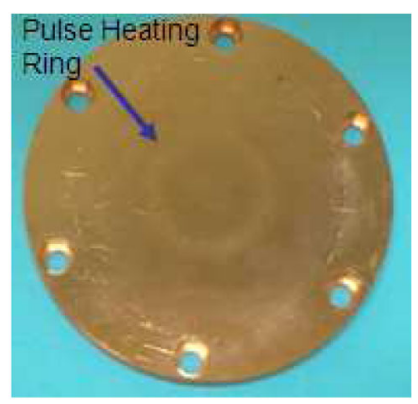

(b)
FIG. 10. Photograph showing copper pulsed heating sample with faintly visible $\mathrm{TE}_{01}$ pulsed heating ring after testing to a pulsed heating temperature of (a) $70^{\circ} \mathrm{C}$ for $4 \times 10^{6} \mathrm{rf}$ pulses and (b) a second $70^{\circ} \mathrm{C}$ run for an additional $4 \times 10^{6}$ rf pulses.

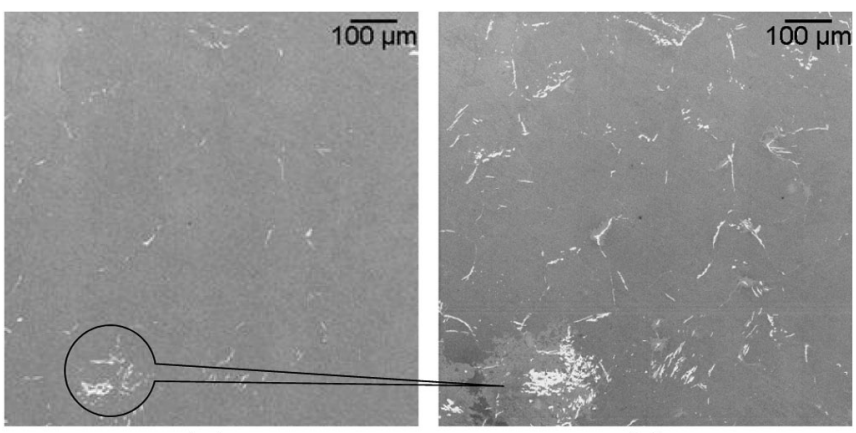

(a)

(b)

FIG. 11. SEM image of copper pulsed heating sample taken at the same location after (a) the first $70^{\circ} \mathrm{C}$ run and after (b) the second $70^{\circ} \mathrm{C}$ run.

$70^{\circ} \mathrm{C}$ run are shown in Fig. 11. Both images were taken at the same location and at the same magnification to compare the level of surface damage between these two $70^{\circ} \mathrm{C}$ runs.

The pulsed heating surface damage included both intergranular and transgranular fatigue cracks within the $\mathrm{TE}_{01}$ pulsed heating ring. Transgranular fatigue cracks are a stochastic process that begins with dislocations that lead to slip lines and slip bands. Repetition of forward and reverse slip results in the initiation of a fatigue cracking which then propagates with an increase in the number of pulses and/or temperature. Intergranular fatigue cracks are microfractures that occur along grain boundaries. An SEM image with both types of fatigue cracks is shown in Fig. 12. The thermal fatigue stresses produced a noticeable slip band extrusion in this sample and in most of the samples tested in this study. After the extended run time at $70^{\circ} \mathrm{C}$, the fatigue crack length and extrusions had increased. An example of the latter is encircled on the image in Fig. 12(b).

A second copper sample was processed to a peak pulsed heating temperature of $110^{\circ} \mathrm{C}$. A photograph of this sample is shown in Fig. 13(a). In this photograph there are two outer concentric rings visible beyond the pulsed heating

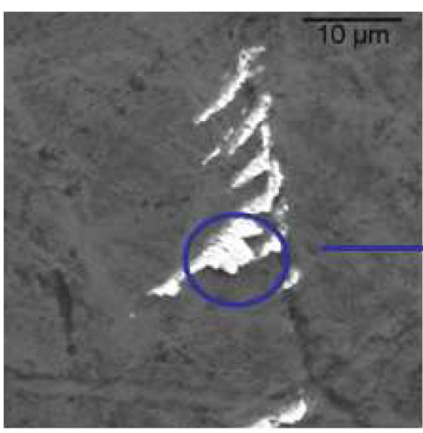

(a)

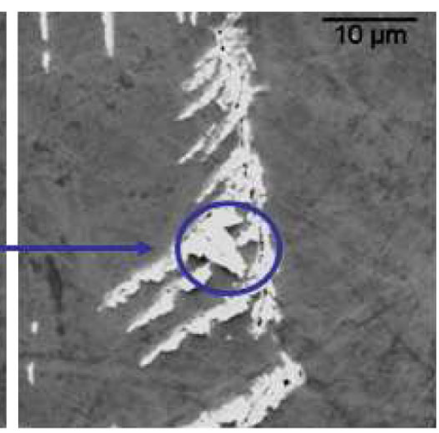

(b)
FIG. 12. SEM image after (a) first $70^{\circ} \mathrm{C}$ run and after (b) second $70^{\circ} \mathrm{C}$ run. Surface extrusions were more severe after extending the rf processing time. 


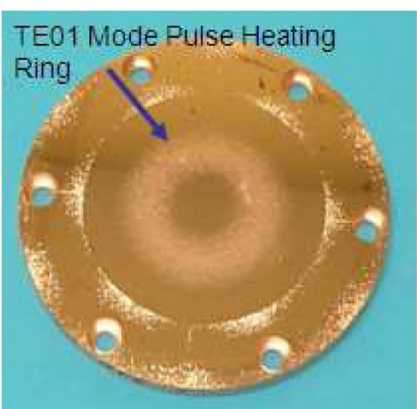

(a)

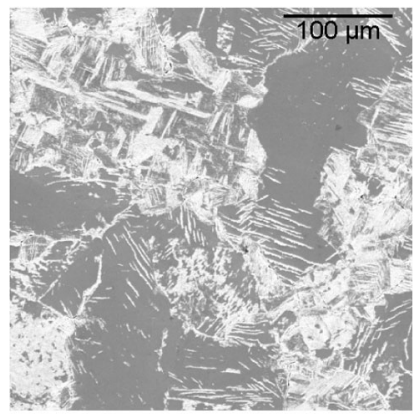

(b)
FIG. 13. Copper pulsed heating sample tested to $110^{\circ} \mathrm{C}$ showing (a) pulsed heating ring and a (b) SEM image showing a typical surface area within the pulsed heating ring at higher magnification.

ring. The first ring is around the outer diameter and the second ring is approximately $1 / 4$ inch past the bolt hole pattern. This may have been the result of gap multipactor due to misalignment between the end plate and the cavity. This problem was corrected prior to testing additional pulsed heating samples.

Like the first copper sample, intergranular and transgranular surface extrusions were evident. The surface damage observed at $110^{\circ} \mathrm{C}$ was significantly more severe compared to the first pulsed heating sample tested to $70^{\circ} \mathrm{C}$. An image showing a typical damaged area on the surface within the pulsed heating ring is shown in Fig. 14(a) and at higher magnification in Fig. 14(b).

The copper sample tested to $110^{\circ} \mathrm{C}$ was cross sectioned through the pulsed heating ring and metallography was conducted to investigate subsurface damage. For comparison, the area just outside of the pulsed heating ring is shown in Fig. 15(a). In this image the grain structure and grain boundaries appear undamaged and the grains extend from 10's to 100's of microns into the depth of the material. A metallography image taken inside the pulsed heating

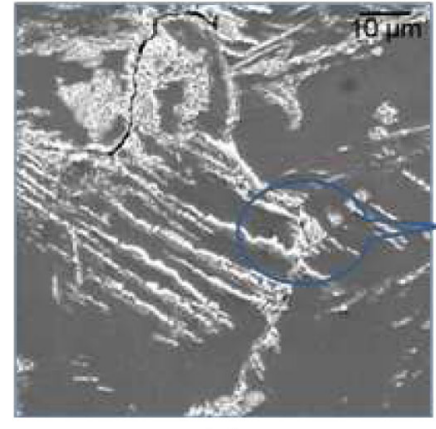

(a)

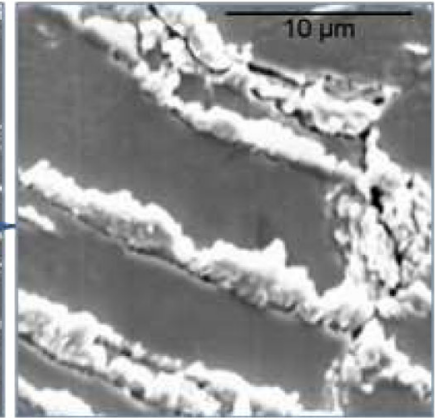

(b)
FIG. 14. SEM images showing an example of the surface damage observed on the copper pulsed heating sample tested to $110^{\circ} \mathrm{C}$ at (a) lower magnification and the same area at (b) higher magnification.

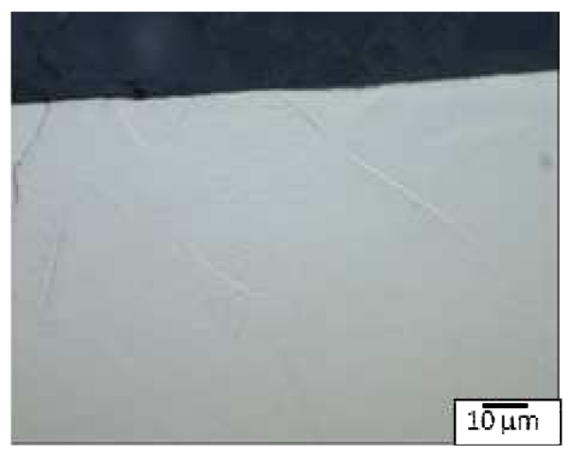

(a)

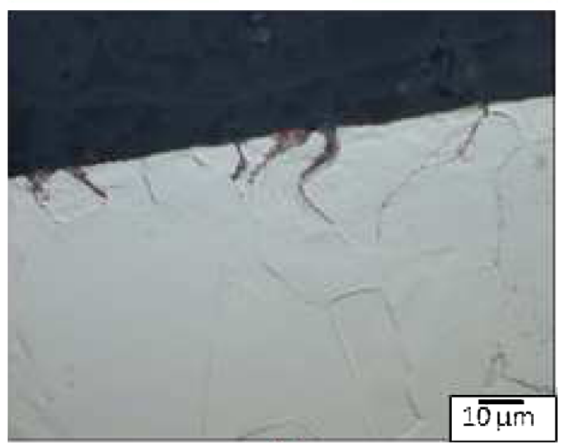

(b)

FIG. 15. Metallography of the copper sample tested to $110^{\circ} \mathrm{C}$. Subsurface images showing (a) outside the pulsed heating ring and (b) within the pulsed heating ring. Grain boundary damage extended $20-40 \mu \mathrm{m}$ below the surface.

ring is shown in Fig. 15(b). Grain boundary separation and damage was clearly evident forming channels that widened near the surface creating a funnel appearance. These areas are responsible for the intergranular extrusions discussed earlier. The channels extend from depths of $20-40 \mu \mathrm{m}$ below the surface. The metallography results suggested only intergranular damage. Internal flaws, inclusions, and grains boundaries are sites where locally the crystal lattice is heavily disturbed. This may facilitate fatigue crack initiation and growth and be the reason why mainly intergranular fatigue cracks have been found.

The next two pulsed heating samples tested were manufactured from $\mathrm{C} 15000$ hot extruded copper zirconium $(\mathrm{CuZr})$. The zirconium content based on the manufacturer's specification for this material is $0.13 \%-0.2 \%$. These samples were machined using diamond fly cutting and were rinsed in alcohol prior to test. Copper zirconium is a copper alloy that has the advantage of maintaining the electrical conductivity of copper but can increase the material strength by the addition of micron size zirconium particles. These particles act as a barrier to dislocation and grain boundary movement which hinders grain growth and retards the recrystallization that leads to material softening.

An SEM image of the CuZr pulsed heating sample taken prior to rf test is shown in Fig. 16. Chemically etching this material led to preferentially etching the softer copper base 


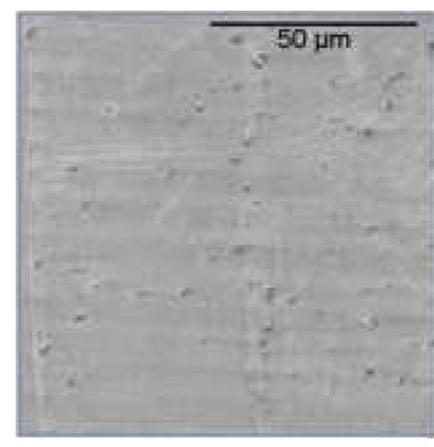

Copper

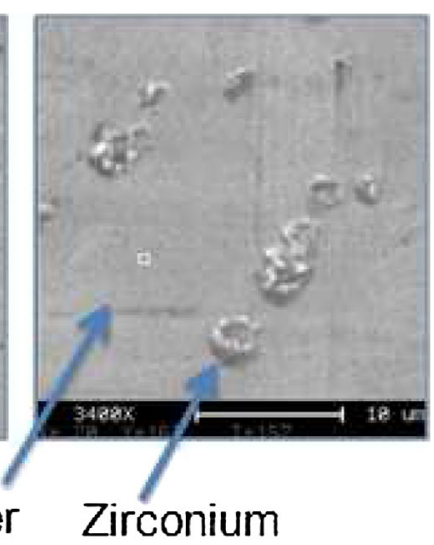

(b)
FIG. 16. SEM image of copper zirconium pulsed heating sample before rf test at (a) low magnification and a section of the same area at (b) higher magnification.

material and resulted in an embedded layer of zirconium particles on the surface. A higher magnification image of these particles that are approximately $1-3 \mu \mathrm{m}$ in size is shown in Fig. 16(b).

A photograph showing the two copper and two $\mathrm{CuZr}$ test samples after test is shown in Fig. 17. On the top row (left), a comparison of the copper sample tested to $70^{\circ} \mathrm{C}$ can be made with the CuZr sample (top row, right) tested to the (a)

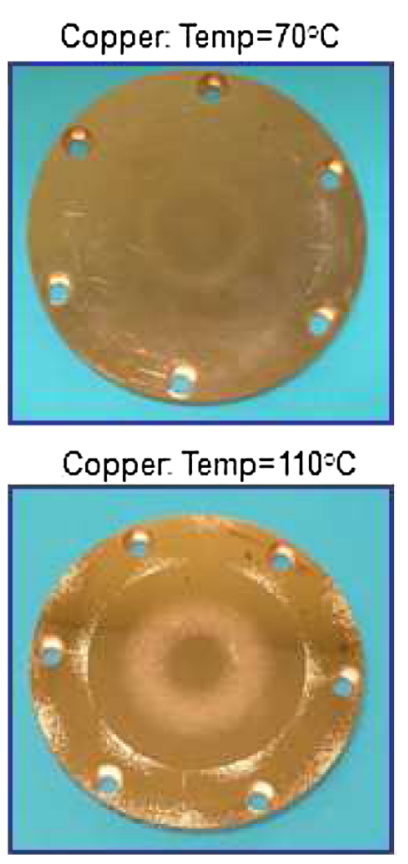

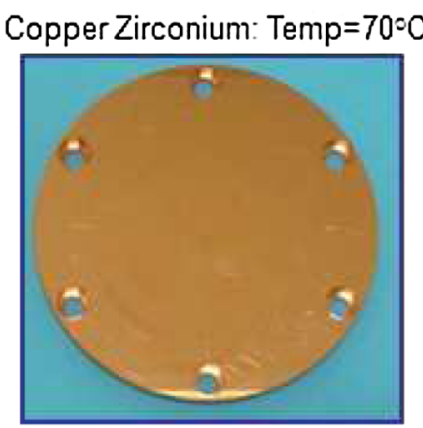

Copper Zirconium: Temp $=100^{\circ} \mathrm{C}$

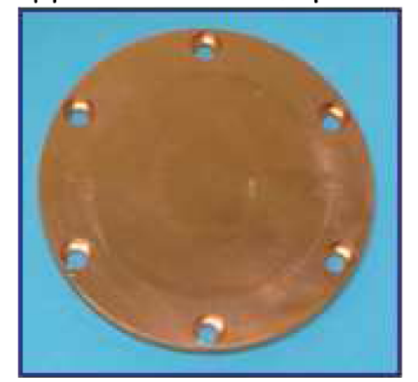

(b)
FIG. 17. Two copper pulsed heating samples tested to a pulsed heating temperature of (a) $70^{\circ} \mathrm{C}$ and $110^{\circ} \mathrm{C}$ and two $\mathrm{CuZr}$ samples tested to (b) $70^{\circ} \mathrm{C}$ and $110^{\circ} \mathrm{C}$. The $\mathrm{CuZr}$ test samples had much less surface damage at a given pulsed heating temperature.

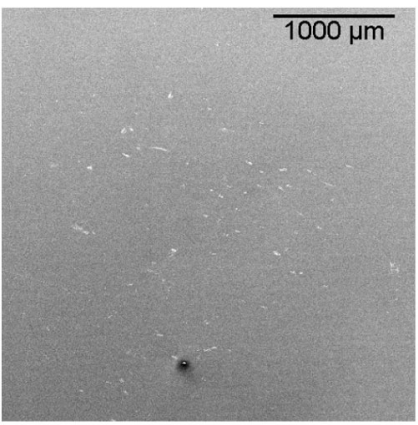

(a)

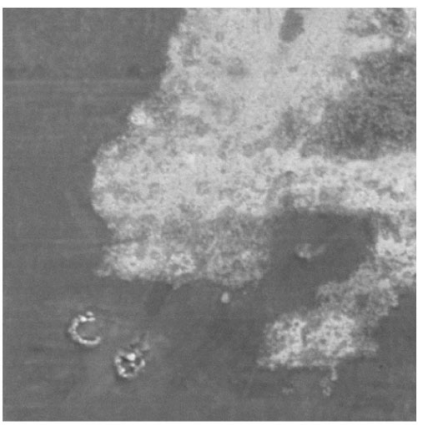

(b)
FIG. 18. SEM image taken inside the CuZr pulsed heating ring after $\mathrm{rf}$ testing to a pulsed heating temperature of $70^{\circ} \mathrm{C}$ showed minor surface damage.

same pulsed heating temperature. The CuZr sample was much less impacted by pulsed heating than the copper sample. At $70^{\circ} \mathrm{C}$ there was minimal microscopic surface damage detected on the CuZr sample.

One section of the pulsed heating ring on the $\mathrm{CuZr}$ sample tested to $70^{\circ} \mathrm{C}$ is shown in Fig. 18(a). The white areas in these images are areas where small amounts of surface melting and surface roughness have occurred. These microscopic specks collectively form the pulsed heating ring. A higher magnification image showing one of these small minimally impacted areas is shown in Fig. 18(b). It was not until reaching a peak pulsed heating temperature of $100^{\circ} \mathrm{C}$ [Fig. 17(b), bottom right, and Fig. 19] that the pulsed heating surface damage on the CuZr sample approached the level of damage observed at $70^{\circ} \mathrm{C}$ on the copper sample. At a pulsed heating temperature of $100^{\circ} \mathrm{C}$, the intergranular and transgranular surface extrusions observed on the $\mathrm{CuZr}$ sample looked similar to

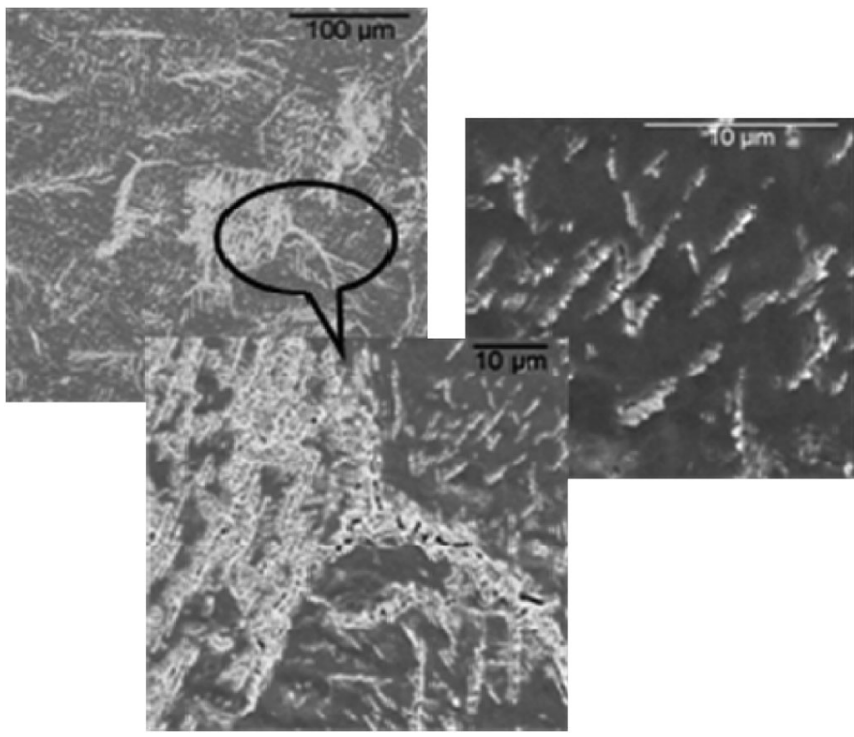

FIG. 19. SEM image taken inside the CuZr pulsed heating ring after $\mathrm{rf}$ testing to a peak pulsed heating temperature of $110^{\circ} \mathrm{C}$. 
the $\mathrm{Cu}$ samples. There was also numerous melted craters located within the pulsed heating ring. Energy dispersive $\mathrm{x}$-ray (EDX) analysis conducted on a random sampling of these sites showed strong zirconium lines inside these craters.

The first four pulsed heating samples discussed so far were preliminary test samples used to validate the operation of the pulsed heating experimental setup and data acquisition system. These samples were also used to establish a testing protocol which was used on the remaining samples tested in this pulsed heating study. All remaining samples discussed in this paper were processed to within $10 \%$ of a pulsed heating temperature of $110^{\circ} \mathrm{C}$ for approximately $10^{7} \mathrm{rf}$ pulses. This requires approximately one week of runtime at a pulse repetition rate of $60 \mathrm{~Hz}$. The physical properties and actual pulsed heating temperatures for each sample can be found in Table I provided at the end of this paper. This study has assumed that the physical properties for the materials tested have remained constant and were not impacted by any of the heat treatment processes.

\section{B. Various pulsed heating samples tested using the established test protocol}

\section{Single-point diamond-turned OFE copper}

The first sample tested using the test protocol was a single-point diamond-turned OFE copper sample that went through a $2 \mu \mathrm{m}$ chemical etch that was followed by hydrogen firing at $1000^{\circ} \mathrm{C}$ for 15 minutes. This fabrication process is similar to steps that are utilized in the fabrication of $\mathrm{rf}$ structures. A photograph of this sample that was taken after testing is shown in Fig. 20. The heat treatment on this sample resulted in a much larger grain size compared to the previous two copper samples tested. Heat treatment can also reduce the material hardness and the fatigue strength which was the case for this sample. After rf testing the $\mathrm{TE}_{01}$ mode pulsed heating ring was strongly pronounced as can be observed in Fig. 20.
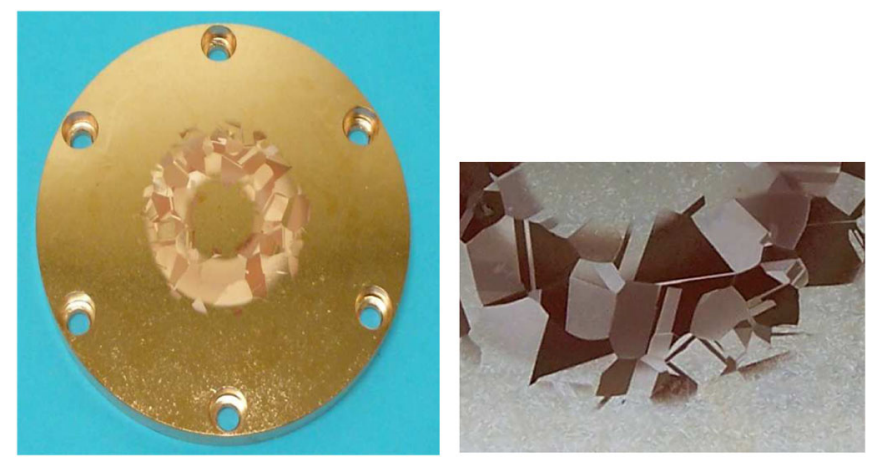

FIG. 20. Diamond-turned OFE copper with larger grains due to hydrogen firing at $1000^{\circ} \mathrm{C}$. Testing to $110^{\circ} \mathrm{C}$ showed pulsed heating surface damage to be dependent on crystallographic orientation.

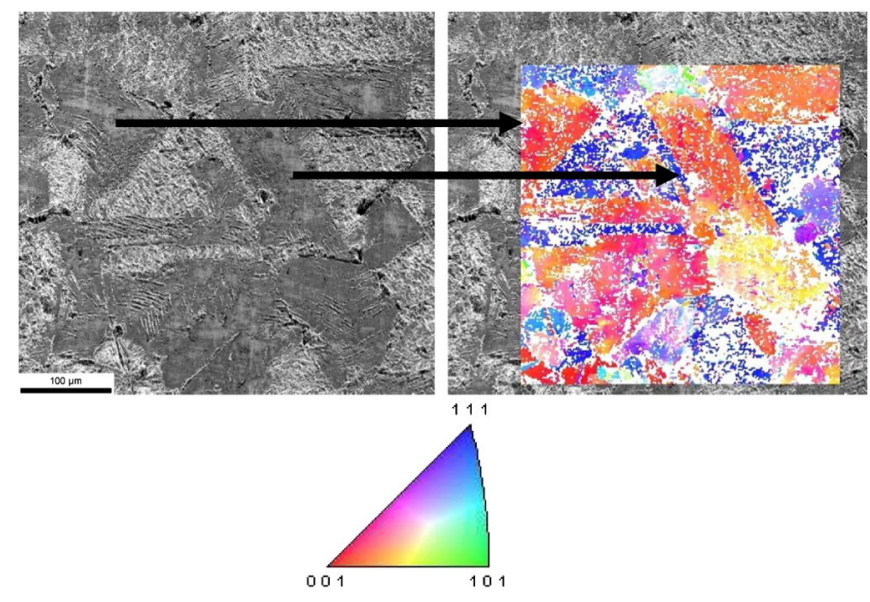

FIG. 21. EBSD image of a copper sample showing pulsed heating surface damage is dependent on crystallographic orientation.

The large grains on this sample provided an opportunity to examine individual grains. SEM analysis showed that some grains were impacted more than other grains within the same radial distance (i.e. same pulsed heating temperature) around the pulsed heating ring. It is shown in [19] that the surface degradation, arising from pulsed heating, is dependent on the crystallographic orientation of the grains with respect to the surface. One of the copper samples was analyzed using electron backscattered diffraction (EBSD). EBSD is a microstructural crystallographic technique used to examine the crystallographic orientation of a material using a scanning electron microscope equipped with a backscattered diffraction camera. An EBSD map showing a segment of the pulsed heating ring of a copper sample is shown in Fig. 21. The SEM images on the left and on the right are the same. The image on the right has a colored overlay on top of the SEM image that provides information on the crystallographic orientation. The out-of-plane orientation is determined by using the color legend that is situated below the SEM images. The arrows have been manually drawn in to point out that the darker regions, which have the least amount of surface damage, correspond to the color grid for a [100] crystal orientation. The grain orientation with the most severe damage (lighter regions) corresponds to a [111] grain orientation. The results of this study suggest that single crystal copper having a [100] orientation should outperform polycrystalline copper under the same pulsed heating conditions. An experiment using [100] single crystal copper was conducted as part of this study and will be discussed later in this paper.

An SEM image showing a twin grain boundary with pulsed heating damage covering the entire surface area of the grain and bounded by a grain which had minimal surface damage is shown in Fig. 22. There were many twin grains on this sample that were observed to show 


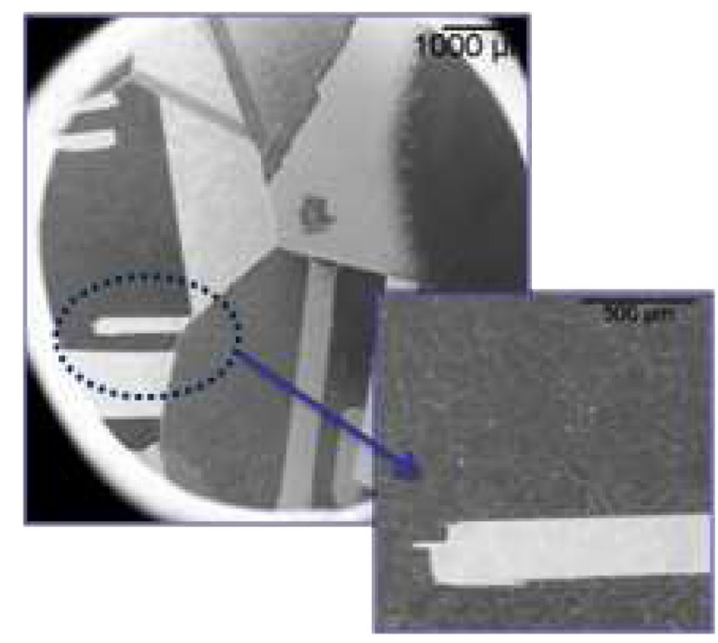

FIG. 22. Twin grains with significant pulsed heating damage surrounded by minimally impacted grains.

excessive damage compared to neighboring grains. Although EBSD has not been conducted on this sample, the previous EBSD results suggest that the darker, less damaged grains are likely to have a [100] crystal orientation.

Analysis on numerous grains has also revealed that not all grains are impacted topographically the same by pulsed heating. One example of this can be seen in Figs. 23 and 24. The SEM image on the left side of these figures shows two grains with the grain boundary running diagonally in the picture. The images on the right of Figs. 23 and 24 are higher magnification images of these two grains near the grain boundary. The pulsed heating damage on the grain shown on the right of Fig. 23 has a matted surface and the damage shown on the right of Fig. 24 has a weavelike pattern. The grain with the matted surface is created by extrusions occurring through submicron size pits that form on the face of the crystal. These micropits do not seem to be associated with slip bands as previously seen on the

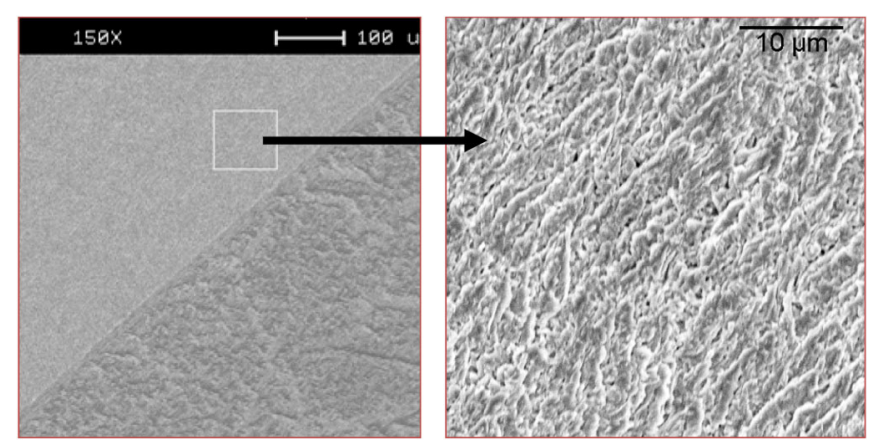

FIG. 23. Two neighboring grains on a copper pulsed heating sample that was tested to $110^{\circ} \mathrm{C}$ showing different types of pulsed heating damage (left image). The top left grain had damage that initiated with micropits and resulted in a matted appearance (right image).

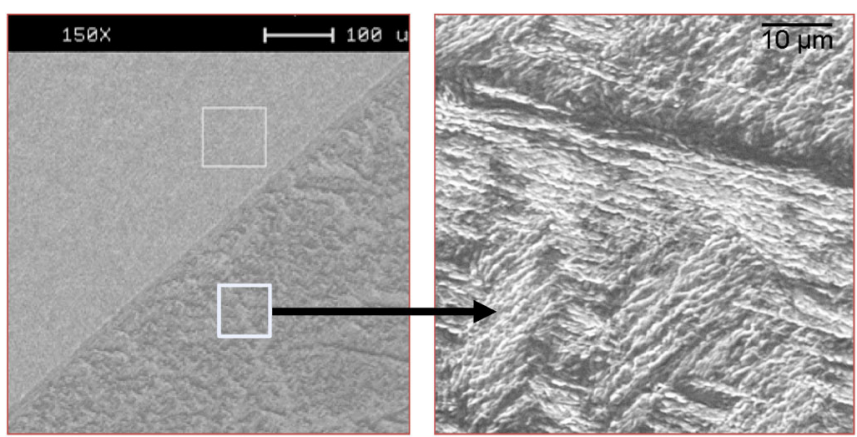

FIG. 24. The bottom right grain has a weavelike appearance that was initiated by intergranular and transgranular fractures.

other test samples and which are often times a precursor to transgranular cracking. The weave pattern is created by the combination of intergranular and transgranular microfractures. The crossing of transgranular cracks propagating perpendicular (or oblique) and parallel to grain boundaries creates the weave-pattern appearance that is observed on the grain of Fig. 24. The grain boundaries serve as a termination point for the propagation of these transgranular fractures. Earlier stages of both the micropits and weavepattern fractures will be shown and discussed later in this paper.

\section{HIP copper}

As part of the study to evaluate large grained materials, two HIP copper pulsed heating samples were tested. Hot isostatic pressing (HIP) is a manufacturing process that subjects the material to both elevated temperature and isostatic gas pressure in a high pressure containment vessel. This process is used to reduce porosity and can improve the material fatigue strength. The two HIP copper pulsed heating samples that were rf tested are shown in Fig. 25. The HIP fabrication process for these samples was conducted at a temperature of $800^{\circ} \mathrm{C}$ and a pressure of $1200-\mathrm{kg} / \mathrm{cm}^{2}$ for two hours in argon gas. The material was then machined using single-point diamond turning. The HIP copper pulsed heating sample shown in Fig. 25(a)

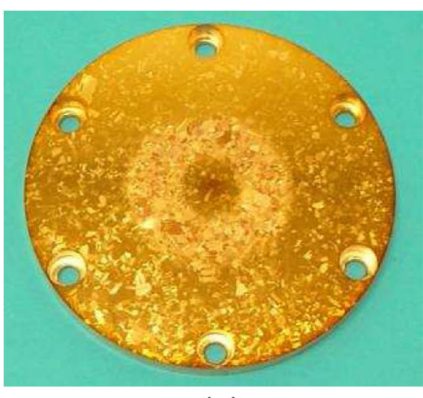

(a)

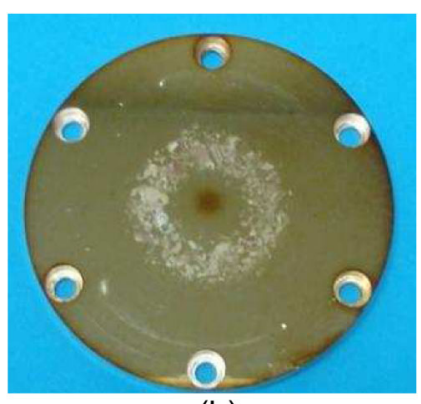

(b)
FIG. 25. Two HIP copper pulsed heating samples. One sample was (a) chemically etched and the other sample was (b) not etched. 
went through a two-micron chemical etch prior to rf test. The etching process, besides removing undesirable protrusions and foreign particulates, removes a different amount of material from the grain boundaries due to a different chemical potential, when compared to the interior of grains, here resulting in a deepened grain boundary. This effect is used in metallography in order to reveal the material microstructure as shown for the sample in Fig. 25(a).

Both of the HIP copper samples were rf processed to a pulsed heating temperature of $110^{\circ} \mathrm{C}$. Both samples had significant pulsed heating damage and there was no major difference between the etched and nonetched samples. On the nonetched sample, the grains which were initially not visible are now visible but only within the pulsed heating ring and is due to the difference in topographical damage on the various grains.

The experimental results for the HIP copper samples were very similar to the large grained, diamond-turned, etched, and hydrogen fired copper sample previously discussed. The pulsed heating crystallographic orientation dependency is evident in Fig. 26 which shows variations in both the degree and type of pulsed heating surface damage on different grains. Again, the darker grains in these images have the least amount of surface damage.

An SEM image showing the outer edge of the pulsed heating ring on the HIP copper sample that was chemically etched is shown in Fig. 27. Because the temperature is lower on the outer edges of the pulsed heating ring, examining the outer edges can reveal the initial impact or onset of pulsed heating surface damage that occurs at lower temperatures. Grain boundaries were observed to be the first locations impacted by pulsed heating. They have a lower energy barrier and are regions where atoms have become misaligned and are susceptible to localized grain boundary defects and foreign particulates.

Individual grains that are located between the outer edge $\left(T_{\min }\right)$ and the center of the pulsed heating ring $\left(T_{\max }\right)$ are

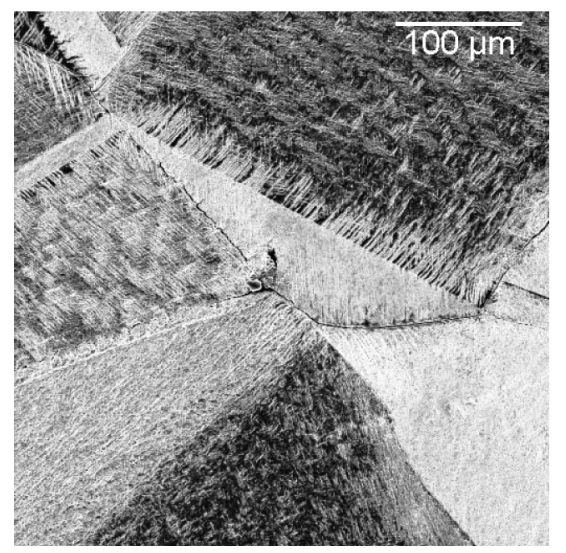

FIG. 26. High magnification SEM image of a HIP copper sample showing variations in the degree and type of pulsed heating surface damage on individual grains.
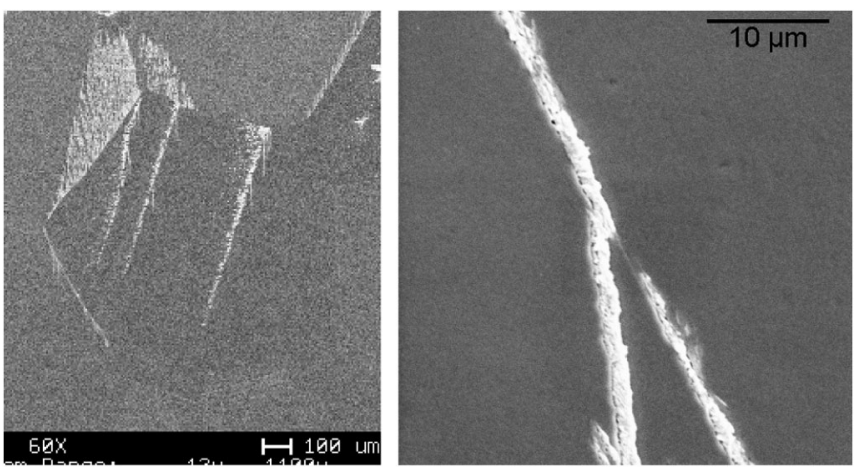

FIG. 27. SEM image showing the outer edge of the pulsed heating ring where grain boundaries are the first to be impacted.
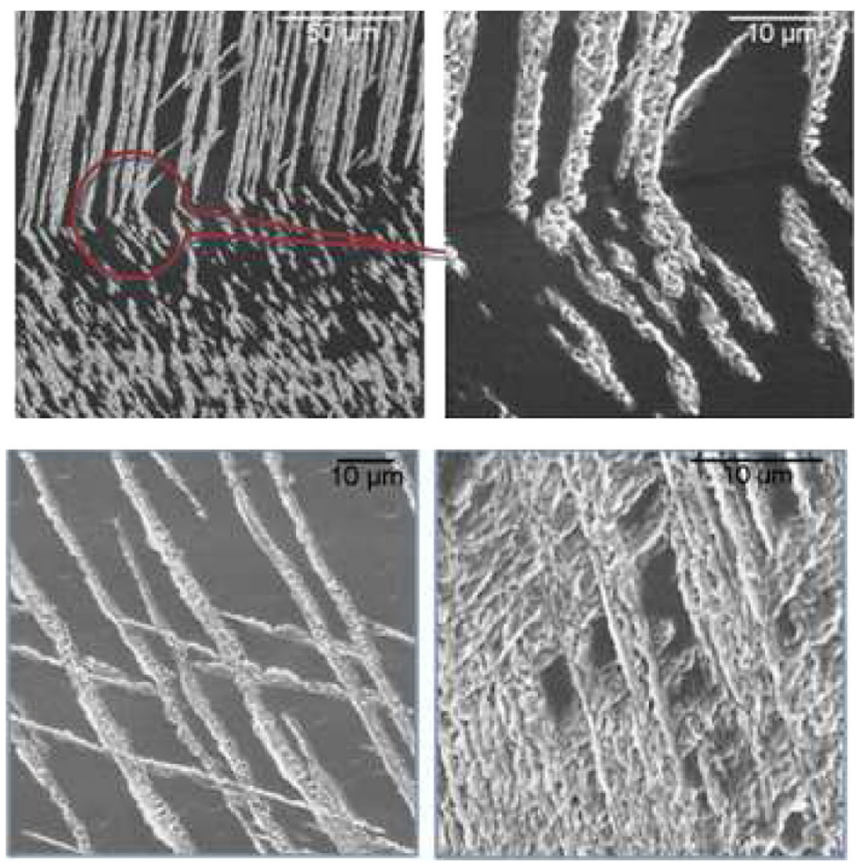

FIG. 28. Transgranular extrusions terminating along grain boundaries (top) and the resultant weavelike pattern (bottom) that is created when they are oriented in different directions.

shown in Fig. 28. The development and orientation of the surface extrusions is evident in these images. The top two images show the extrusions terminating along grain boundaries and the bottom images show the resultant weavelike pattern that is created when the transgranular extrusions are oriented in different directions.

\section{Electrodeposited copper}

An electrodeposited copper pulsed heating sample was also tested in this study. The substrate was C10100 OFE grade copper. The electrodeposition process parameters control the grain size and grain morphology which ultimately determines the strength and ductility of the copper. A photograph of the electrodeposited copper sample taken 


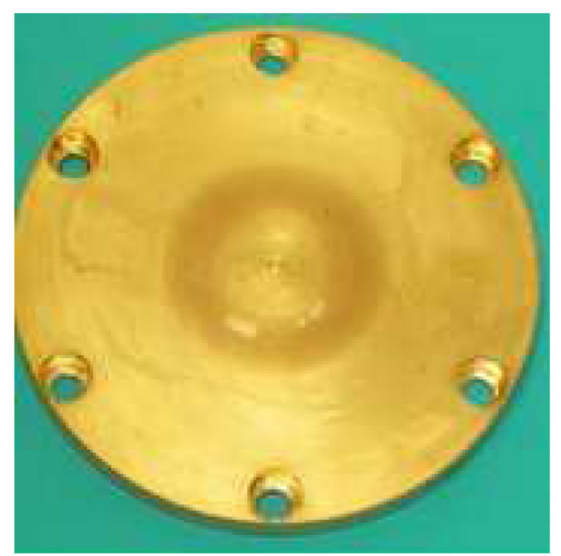

FIG. 29. Photograph of electrodeposited copper sample after processing up to a pulsed heating temperature of $110^{\circ} \mathrm{C}$.

after testing to a pulsed heating temperature of $110^{\circ} \mathrm{C}$ is shown in Fig. 29. The plating thickness was approximately $300 \mu \mathrm{m}$ and was deposited onto the copper substrate using a pyrophosphoric acid copper plating technique. No heat treatment was utilized on this sample.

At the end of the plating process the sample was machined using diamond turning which was followed by a chemical etch. In the absence of polishing or using a brightener process, electrodeposition often forms a matte finish as is the case with this sample. After rf testing the electrodeposited copper sample to a pulsed heating temperature of $110^{\circ} \mathrm{C}$, the pulsed heating ring was clearly visible (Fig. 29). Elongated dark patchy areas were observed on this sample and were also commonly seen on other samples that had similar material hardness values. This nonuniformity is more evident in Fig. 30.

The darker locations in the SEM images are areas that had less surface damage compared to the nearby surrounding regions. The top right image of Fig. 30 gives an

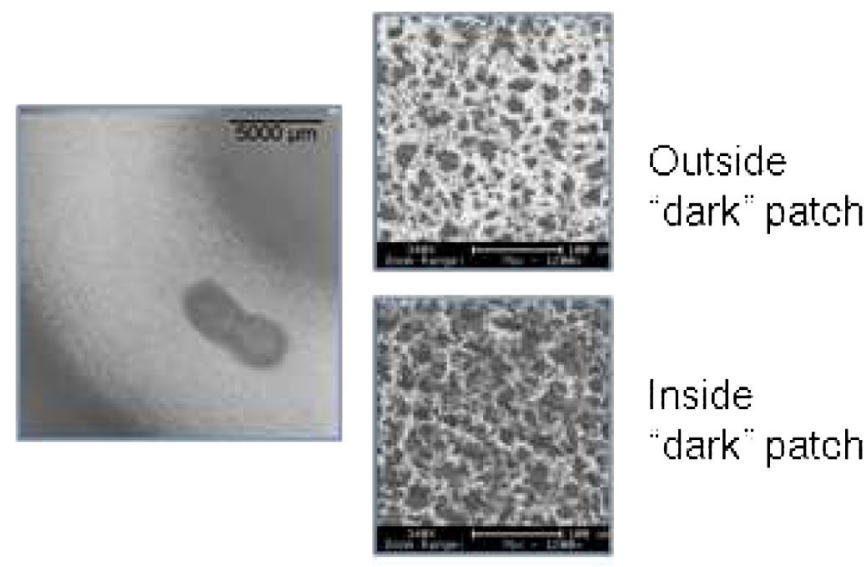

FIG. 30. Nonuniform pulsed heating surface damage. Dark elongated patchy areas were observed inside the pulsed heating ring. These were areas that had less surface damage relative to the rest of the pulsed heating ring.
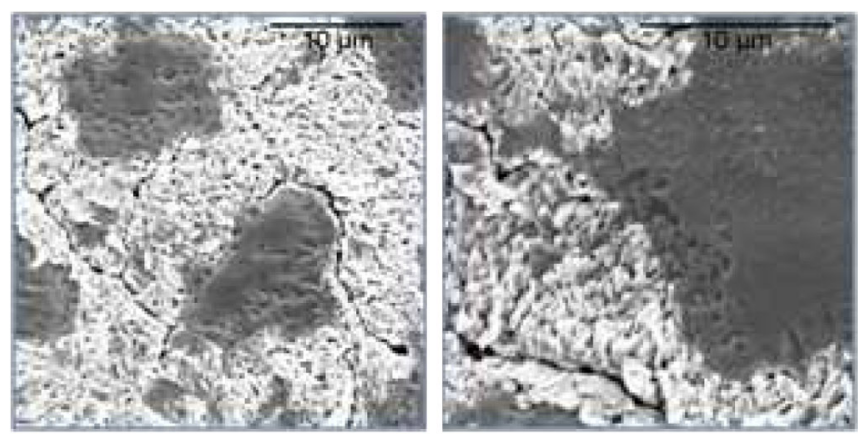

FIG. 31. High magnification SEM image of the electrodeposited copper sample taken after the test. These images show a region inside the dark patchy area.

example of what the surface characteristically looked like within the pulsed heating ring. The bottom right image of Fig. 30 shows what the surface looked like inside the dark patchy area. The damage in both of these regions looks similar but the darker regions are spots on the surface that have been less impacted by pulsed heating. This is easier to see in the high magnification SEM images shown in Fig. 31. The different types of pulsed heating surface damage discussed so far have been due to the transgranular surface extrusions oriented in different directions and terminating on grain boundaries and the damage observed due to extrusions through micropits. In this sample, the pulsed heating damage followed the latter case. The formation of micropits is initiated near grain boundaries and as the pulsed heating temperature increases the number of pits increase and progressively develop further away from the grain boundaries until the remaining grain has been damaged. The micropit extrusions give a stringy or matted surface appearance that has been observed on many of the samples tested. It is speculated that the observed differences in types of pulsed heating damage may be due to a difference in crystallographic orientation.

\section{Single crystal copper [100]}

Earlier it was mentioned, based on the EBSD results, that single crystal copper with a [100] crystal orientation should outperform polycrystalline copper under the same pulsed heating conditions. A preliminary test using a [100] single crystal copper sample was conducted as part of this study and a photograph of the sample is shown in Fig. 32. This sample was fabricated using a 50-mm diameter single crystal copper disk that was glued onto a $75-\mathrm{mm}$ diameter copper substrate with a $50-\mathrm{mm}$ counter bore. The single crystal copper disk was fitted into the counter bore and glued to the substrate using an indium gallium alloy that was heated to a low temperature of $<20^{\circ} \mathrm{C}$. The single crystal copper was polished using chemical mechanical polishing techniques.

This sample was initially processed to a pulsed heating temperature of $70^{\circ} \mathrm{C}$ [Fig. 32(a)] for approximately one 

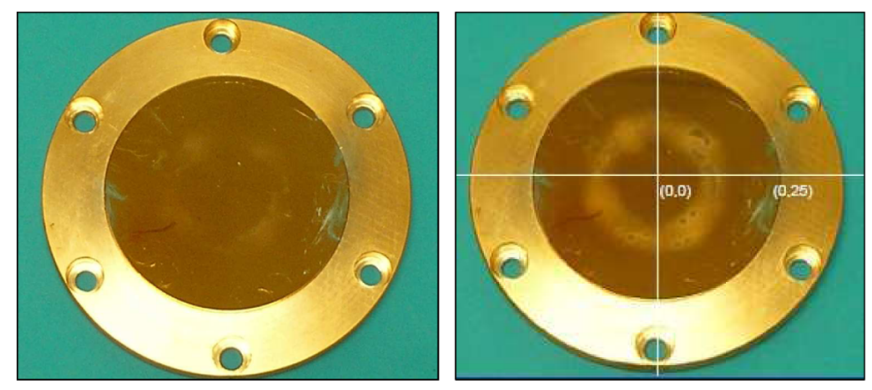

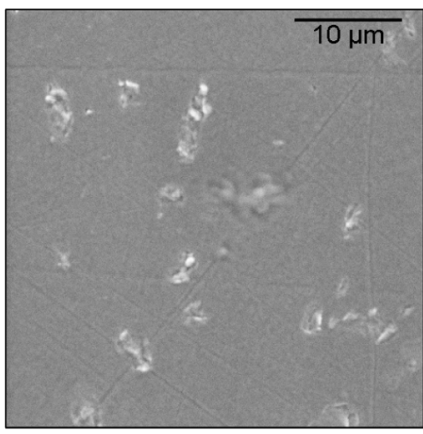

(a)

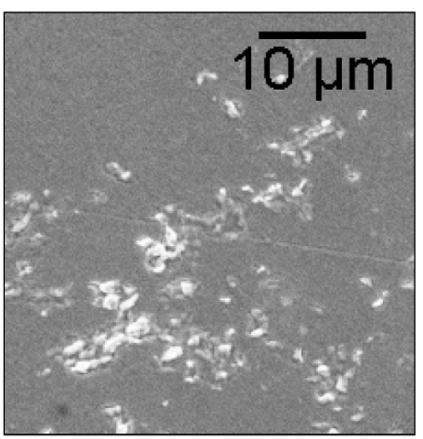

(b)
FIG. 32. Single crystal copper with [100] grain orientation after rf testing at a pulsed heating temperature of (a) $70^{\circ} \mathrm{C}$ and (b) $110^{\circ} \mathrm{C}$.

day of operation and then temporarily removed from the cavity for a preliminary SEM inspection. The pulsed heating ring was already faintly visible. This can be seen in the top left photograph of Fig. 32. The SEM image in the bottom left shows a typical area within the pulsed heating ring after the initial $70^{\circ} \mathrm{C}$ test. The surface damage observed was minimal and appeared as small pits. There were no microfractures located on the surface as a result of this initial $70^{\circ} \mathrm{C}$ test run. The photograph in the top right of Fig. 32 is the same sample after reinstalling it into the cavity and continuing with the established test protocol. At $110^{\circ} \mathrm{C}$ there was a significant increase in surface damage and transgranular slip band extrusions (Fig. 33). The lengths of the extrusions and fractures were much smaller than in the previous samples tested and were typically
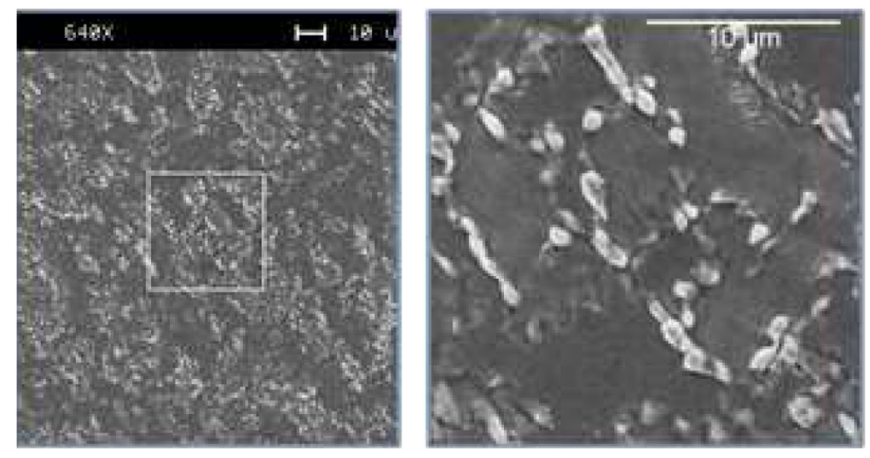

FIG. 33. At $110^{\circ} \mathrm{C}$ some areas on the single crystal copper showed evidence of microfractures and extrusions.
$<10 \mu \mathrm{m}$. The depth of the transgranular extrusions appear shallow and may be due in part to the absence of grain boundaries. This test did not suggest that grain orientation alone was sufficient to overcome surface fatigue issues associated with rf pulsed heating.

\section{Hardness analysis on single crystal [100] copper}

After the rf testing was completed, the single crystal copper sample was shipped to KEK for hardness testing. The hardness measurements were performed with a microhardness tester equipped with a Vickers diamond pyramid. The unit of hardness is the Vickers pyramid number (HV). This test determines the material's ability to resist plastic deformation and a higher HV number indicates a harder material.

A graph showing the hardness values measured on the surface along the radial axis is shown in Fig. 34 and is superimposed on the single crystal test sample. The data points beginning at the center of the sample and designated as $0-\mathrm{mm}$ on the graph extends radially outwards for a distance of $25 \mathrm{~mm}$. This distance is approximately the radius of the single crystal copper insert. The plot shows that work hardening occurred in the pulsed heating ring and that the measured material hardness increased nearly proportionally to the pulsed heating temperature. The graph of radial distribution of hardness and cyclic temperature rise versus radial position (top right of Fig. 34) showed that approximately $60 \mathrm{~K}$ has to be overcome to cause surface hardening.

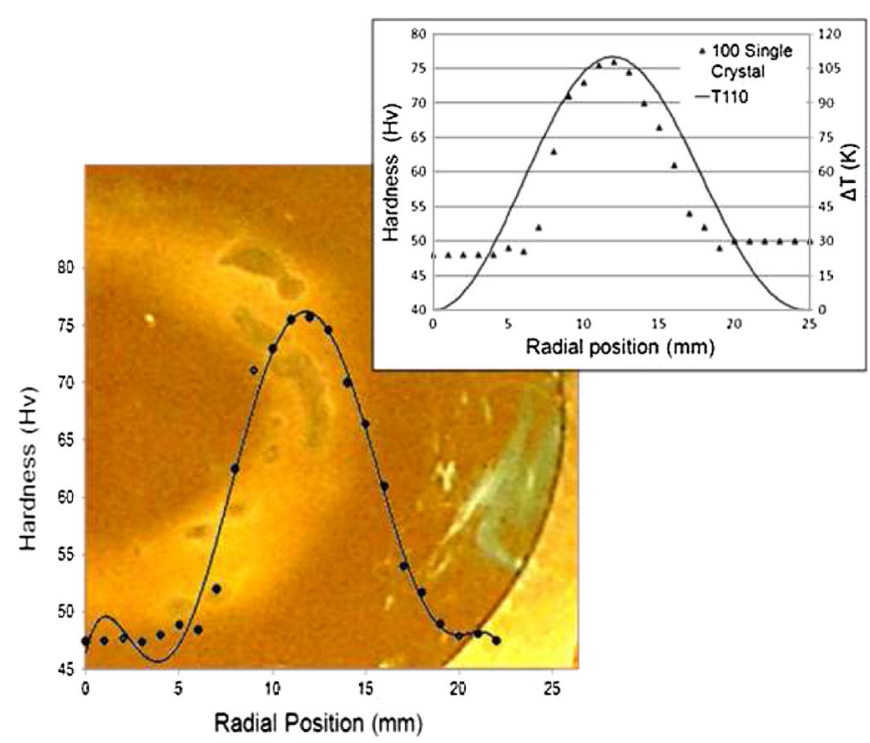

FIG. 34. Vickers hardness test results on [100] single crystal copper after the rf test showed work hardening occurred on the surface within the pulsed heating ring. Radial distribution of hardness and cyclic temperature rise shows that a threshold of approximately $60 \mathrm{~K}$ has to be overcome to initiate surface hardening. 


\section{Copper chromium}

As a continuation of the copper alloy study, two copper chromium pulsed heating samples were tested. They were manufactured from C18200 OFE chromium copper stock. They were machined using a standard carbide cutting tool and afterwards were cleaned and then chemically etched for 30 seconds. A photograph showing both of these samples is shown in Fig. 35. These photographs were taken after they were both tested using the established test protocol. Prior to the test, the sample on the right was subjected to a $988^{\circ} \mathrm{C}$ vacuum braze cycle. This included a $12 \mathrm{~h}$ ramp-up time, 5 min steady state, followed by overnight cooling in vacuum. The melting temperature of chromium copper is approximately $1075^{\circ} \mathrm{C}$ and, while the vacuum braze cycle was below this temperature, the discoloration observed on the surface is likely due to the melting of micron size chromium particles that were embedded on the surface prior to heat treatment. This was also observed on a copper chromium test piece subjected to a dry hydrogen heat treatment at approximately the same temperature (Fig. 36).

The photograph and SEM image at the top of Fig. 36 were taken prior to any heat treatment. The color of the test piece (top left) looked similar to copper and in the SEM image (top right) the dark areas are copper and the white areas are chromium particles. The geometry of the embedded chromium particles on the surface was typically either elongated or spherical before any heat treatment. After heat treatment, the elongated particles were no longer observed on the surface and surface analysis of the areas that were copper prior to heat treatment now showed much higher levels of chromium. After heat treatment the test piece was discolored and had a grayish tint which can be seen in the bottom photograph and SEM image of Fig. 36.

The discoloration on the heat treated test piece was consistent with that observed on the heat treated $\mathrm{CuCr}$

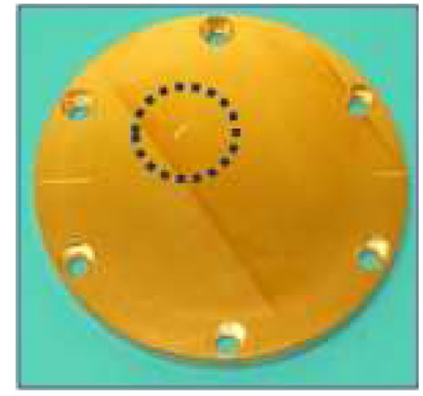

(a)

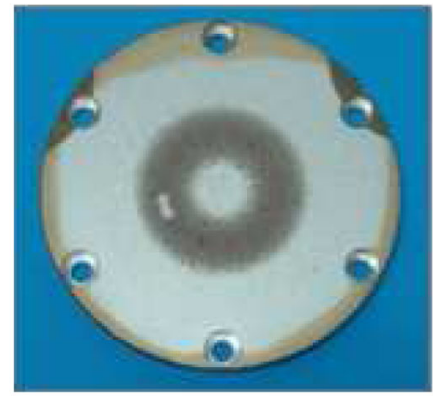

(b)
FIG. 35. Two copper chromium pulsed heating samples that were tested to a pulsed heating temperature of $110^{\circ} \mathrm{C}$. (a) Nonheat treated sample had minimal surface damage and (b) heat treated sample that went through a $988^{\circ} \mathrm{C}$ vacuum braze cycle had significantly more pulsed heating surface damage than the nonheat treated sample.

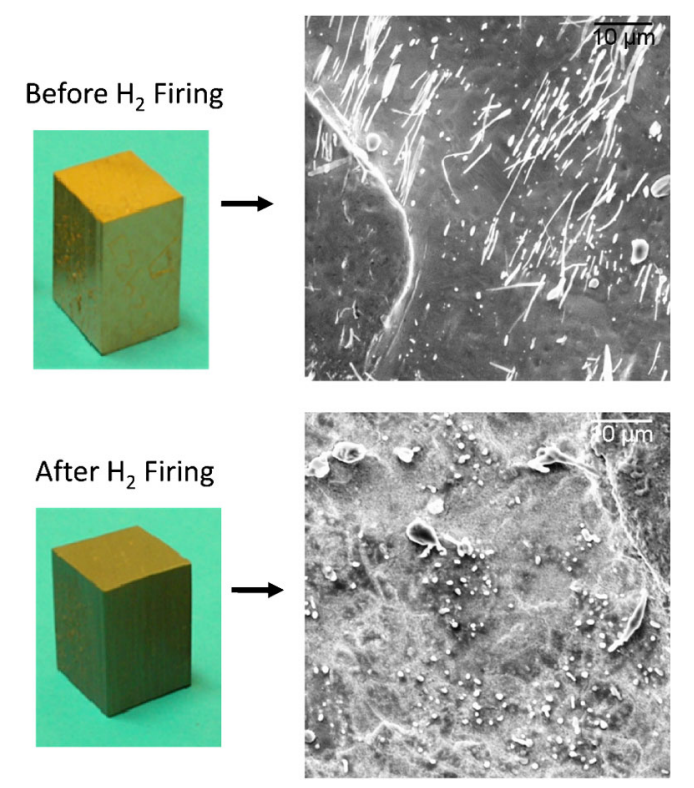

FIG. 36. Chromium copper test piece before (top) and after (bottom) dry hydrogen firing.

pulsed heating sample. The pulsed heating ring on the softer heat treated $\mathrm{CuCr}$ sample is clearly evident in Fig. 35(b). It had significantly more pulsed heating surface damage than the one without the heat treatment. On the nonheat treated sample only a small patch of surface damage was visibly evident and is encircled in the photograph of Fig. 35(a). At higher magnification, a pulsed heating ring was observed but only transgranular extrusions were found. In Fig. 37(a), the large number of grain boundaries that were impacted can be seen in this lower magnification SEM image. The grain boundaries appear whiter than the surrounding surface area. The SEM image in Fig. 37(b) provides a closer view of one of these impacted grain boundaries. The grain boundary extrusions appear white in the image and the white specks that can be seen randomly scattered over the surface are the submicron size embedded chromium

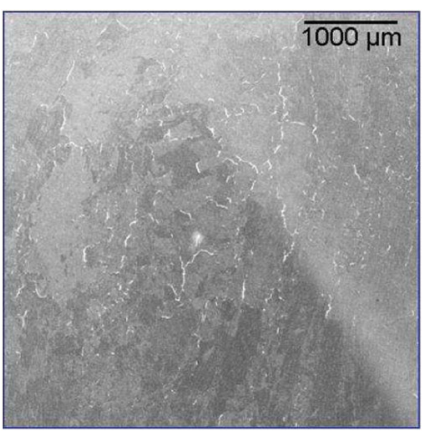

(a)

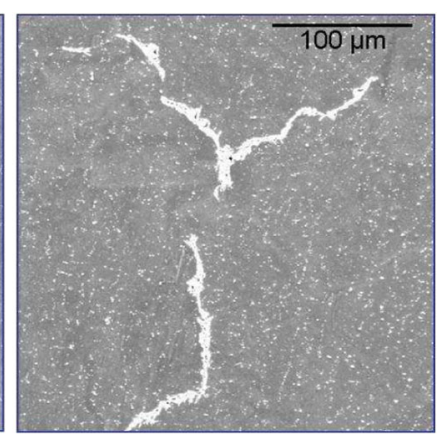

(b)
FIG. 37. SEM image of nonheat treated copper chromium sample. Grain boundaries were the locations primarily impacted on this sample. 
particles. They appear white in the images because they protrude from the surface and emit more secondary electrons which leads to a brighter contrast in the scanning electron microscope.

\section{Copper zirconium}

Cold worked copper zirconium (CuZr) and annealed copper zirconium were tested. One advantage of cold working a material is that it increases the material hardness. It is known that annealing a material increases the grain size and softens many metals. A photograph showing both the cold worked $\mathrm{CuZr}$ and the annealed CuZr sample is shown in Fig. 38. Both of these photographs were taken after rf processing using the test protocol. The larger grains on the annealed $\mathrm{CuZr}$ are evident in the photograph of Fig. 38(b). The softer annealed sample has a significantly more pronounced pulse heating ring in contrast to the harder cold forged CuZr sample [Fig. 38(a)].

The annealed sample was manufactured from C15000 hot extruded copper zirconium that was machined using diamond fly cutting and then cleaned and heat treated prior to test. The heat treatment consisted of a $985^{\circ} \mathrm{C}$ vacuum braze cycle. This included a $12 \mathrm{~h}$ ramp-up time, $5 \mathrm{~min}$ steady state, followed by overnight cooling in vacuum. The cold worked sample was manufactured using C15000 copper zirconium that was solution heat treated. The cold working process used swaging; a forging process where the dimensions are altered using a die. The initial diameter of the copper zirconium ingot was $50 \mathrm{~mm}$ and the diameter of the pulsed heating samples after forging were approximately $76 \mathrm{~mm}$. Cold working makes the grain size smaller. After the swaging process the sample was precipitation hardened and then machined using diamond fly cutting. The sample was cleaned but not etched prior to test.

SEM analysis of the cold worked CuZr sample prior to rf test showed that the surface had numerous pits on the surface (Fig. 39). These pits are most likely caused by embedded zirconium particles that were freed from the surface during the fabrication process. Remarkably, at a

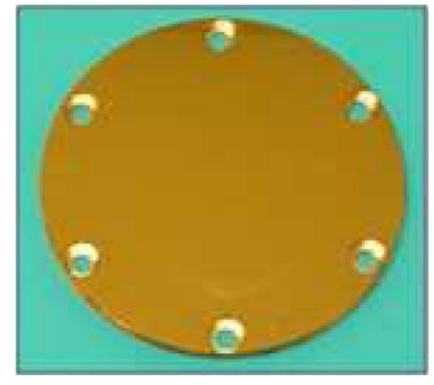

(a)

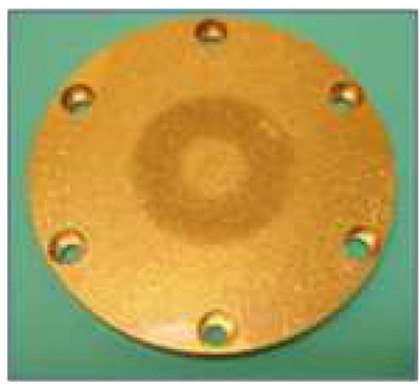

(b)
FIG. 38. (a) Cold worked copper zirconium and (b) annealed copper zirconium after rf testing. The harder material (cold worked) had significantly less pulsed heating surface damage compared to the softer annealed $\mathrm{CuZr}$.
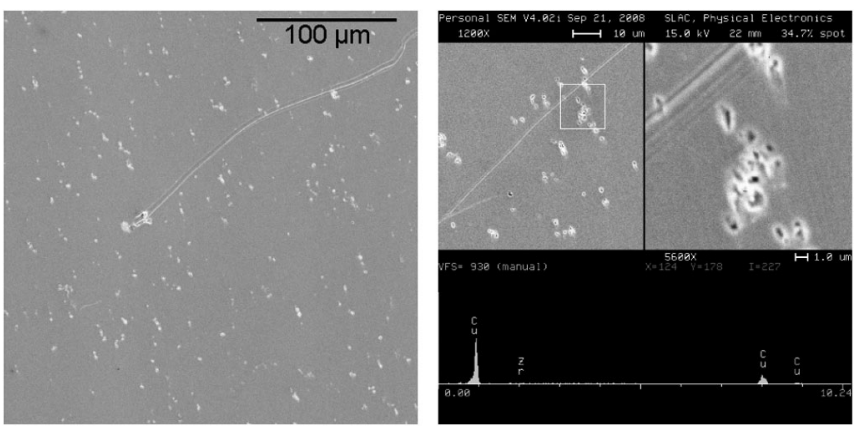

FIG. 39. Cold worked $\mathrm{CuZr}$ sample before test showing surface pits which are likely due to embedded zirconium particles ejected during the fabrication process.

pulsed heating temperature of approximately $110^{\circ} \mathrm{C}$, there was no clear evidence of pulsed heating surface damage on this sample; however, early stages of surface damage may have been masked by the numerous surface pits. Since no discernible surface damage was detected, the cold worked $\mathrm{CuZr}$ sample was reinstalled into the cavity and the power was increased until a pulsed heating temperature of approximately $150^{\circ} \mathrm{C}$ was obtained.

The photograph in Fig. 40 shows the CuZr sample after testing to approximately $150^{\circ} \mathrm{C}$. At this temperature, the pulsed heating ring was faintly visible. A low magnification SEM image showing one region of the pulsed heating ring after rf testing is shown in Fig. 41.

The light colored spots in Fig. 41 are areas where very small microscopic surface damage occurred. This can be seen more clearly in the higher magnification images of Fig. 42 which show microfractures that are typically $<50 \mu \mathrm{m}$ in length. From this experiment, as well as in the other copper alloy experiments that contained both soft and hard materials, the harder materials (nonannealed) substantially outperformed the softer materials. Although this may have been a likely outcome, it is problematic in the development of high-power rf components that typically require a high temperature brazing cycle.

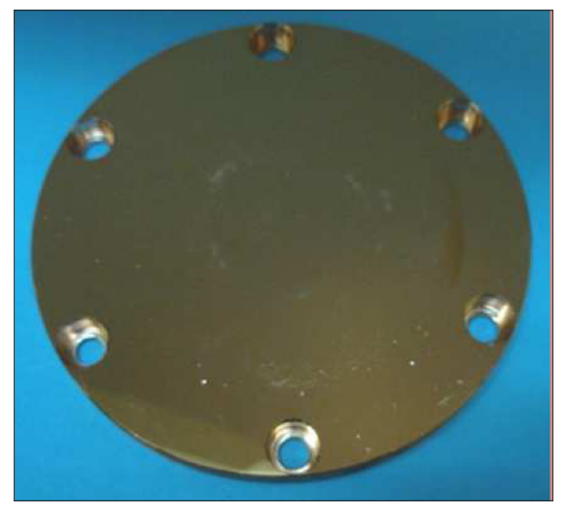

FIG. 40. Photograph of cold worked CuZr sample after testing to a pulsed heating temperature of approximately $150^{\circ} \mathrm{C}$. 


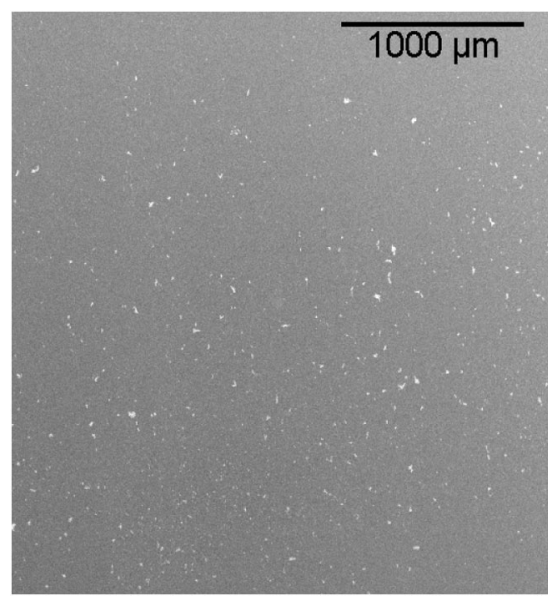

FIG. 41. SEM image showing one section of the pulsed heating ring on the cold worked $\mathrm{CuZr}$ sample after testing to approximately $150^{\circ} \mathrm{C}$.
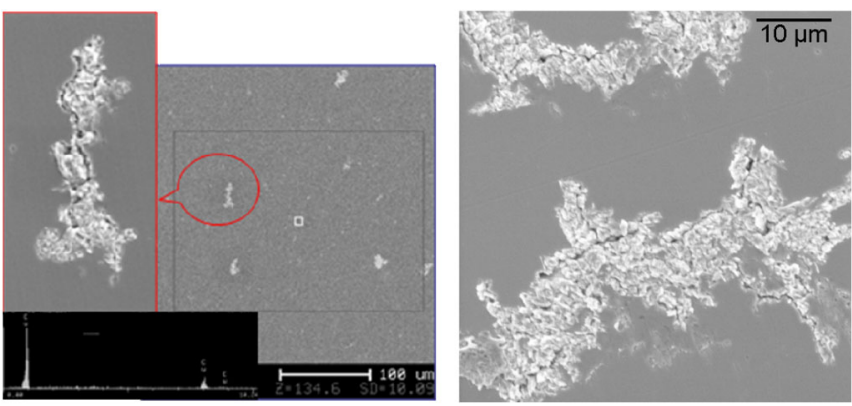

FIG. 42. SEM images showing areas of microscopic surface damage observed on $\mathrm{CuZr}$ sample that was tested to $150^{\circ} \mathrm{C}$. Small fracture lines due to thermal fatigue are now evident.

\section{Copper silver}

Another copper alloy, copper silver $(\mathrm{CuAg})$ was tested in this pulsed heating study. Two $\mathrm{CuAg}$ samples were tested and neither one of them were subjected to heat treatment prior to the rf test. One sample was fabricated by SLAC and the other sample was fabricated by KEK. Like the previous samples, they were tested using the established pulsed heating temperature protocol. Photographs taken of both these samples after testing are shown in Fig. 43. Both samples were fabricated from C10700 stock. The SLAC fabricated sample is shown on the left in Fig. 43(a). The material for this sample was received from the vendor in a mill hardened state. It was machined using standard carbide tooling and afterwards cleaned but not etched.

The pulsed heating ring is faintly visible in the photograph. In order to capture the pulsed heating ring using a digital camera required reducing the overhead illumination sufficiently that the material appears gray in the photograph. The actual color is very similar to the KEK

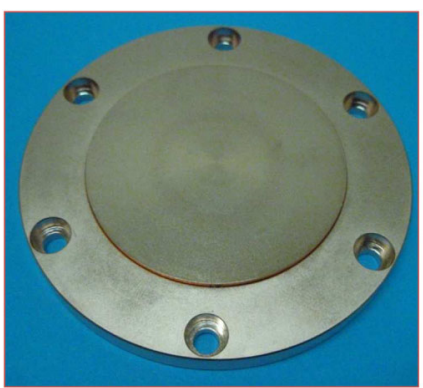

(a)

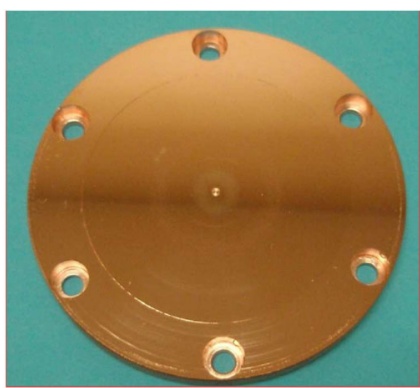

(b)
FIG. 43. A (a) SLAC and (b) KEK fabricated copper silver pulsed heating sample that were tested to $110^{\circ} \mathrm{C}$. Neither of these samples was subjected to heat treatment.

fabricated CuAg sample shown in Fig. 43(b). A low magnification SEM image showing approximately one quadrant of the pulsed heating ring from the SLAC fabricated sample is shown in Fig. 44. Although the pulsed heating ring was visually difficult to see on this sample, it was very evident when viewed with the SEM. The measured hardness value of the $\mathrm{CuAg}$ sample was very close to the $\mathrm{CuCr}$ sample and in comparison the experimental results were very similar. Like the $\mathrm{CuCr}$ sample, the pulsed heating surface damage was dominant along the grain boundaries as shown in Fig. 45.

The second copper silver sample tested that was fabricated by KEK [Fig. 43(b)] was machined using diamond turning. Prior to the test, the sample went through an ultrapure water Megasonic rinse for 1 minute which was followed by a one-minute alcohol rinse in a $40^{\circ} \mathrm{C}$ bath. Similar to the SLAC CuAg test sample, the pulsed heating ring on this sample is faintly visible on the photograph of Fig. 43(b) and surface extrusions were evident. A low magnification image of the pulsed heating ring is shown in Fig. 46. The grain boundaries on this sample were not clearly identifiable like in the SLAC CuAg test sample. Often times it is difficult to locate grain boundaries on

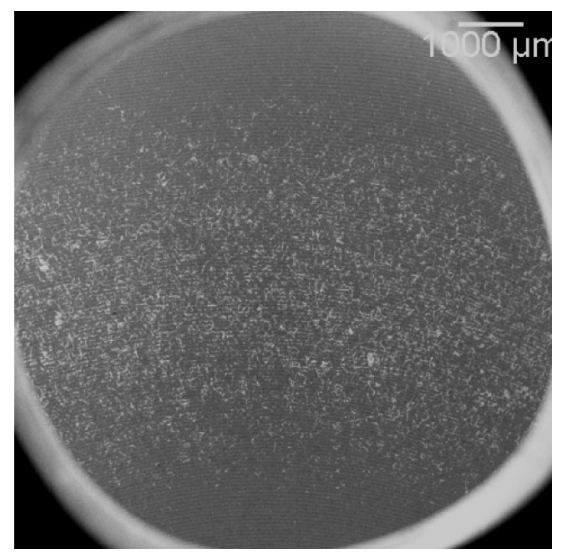

FIG. 44. Approximately one quadrant of the pulsed heating ring of the SLAC fabricated $\mathrm{CuAg}$ sample after rf testing. 
materials that have not been exposed to heat treatment or have not been chemically etched. Numerous fractures were observed on the surface and many were observed to form nearly closed boundaries suggesting that these locations may be grain boundaries [Fig. 46(b)]. The surface damage is shown at higher magnification in Fig. 47.

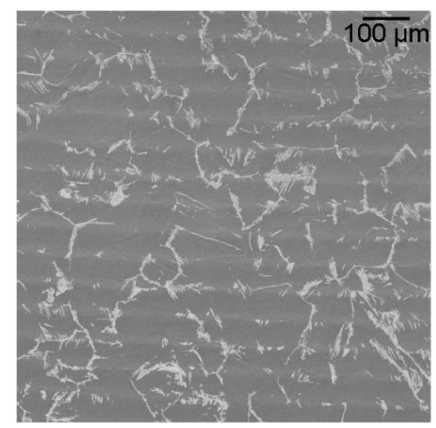

(a)

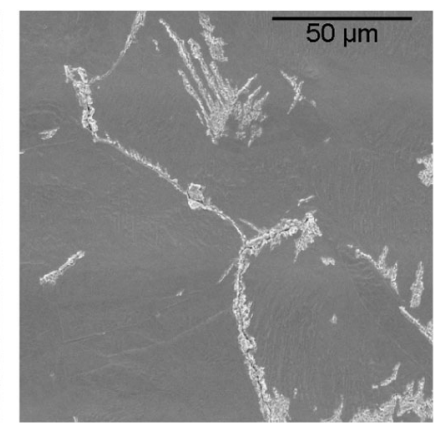

(b)
FIG. 45. High magnification image of the SLAC CuAg pulsed heating sample shown photographically in Fig. 43(a). Pulsed heating damage was dominate along grain boundaries.

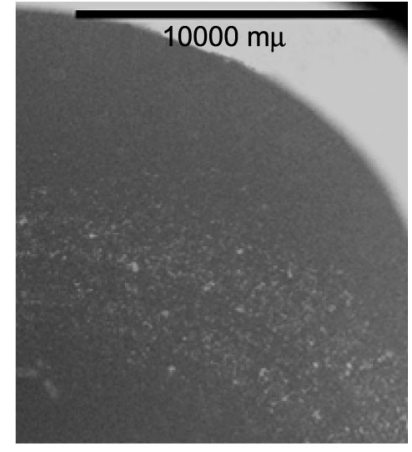

(a)

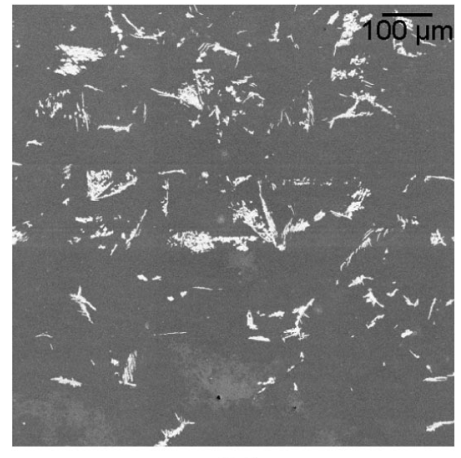

(b)
FIG. 46. Low magnification SEM image of KEK fabricated $\mathrm{CuAg}$ pulsed heating sample.

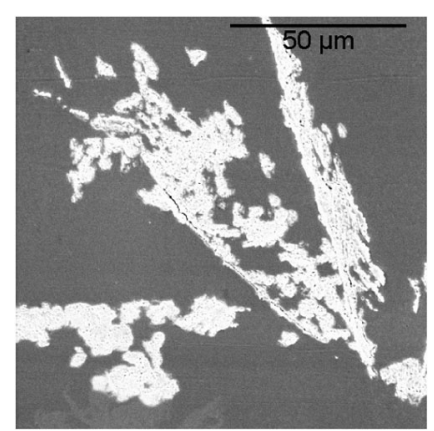

(a)

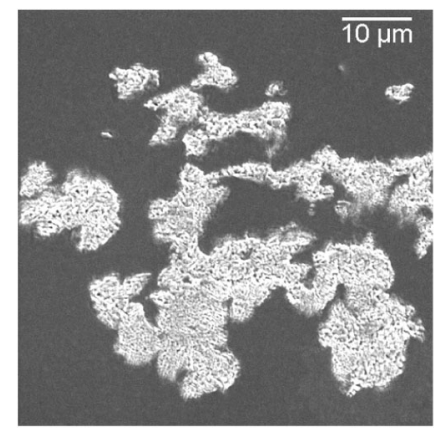

(b)
FIG. 47. High magnification image of KEK CuAg sample showing surface damage (a) along side fracture lines and (b) and on the crystal face.

\section{Silver plated copper}

After the CuAg samples were tested, a silver plated copper pulsed heating sample was installed into the cavity. The plating thickness was approximately 300 microns. After the plating process the sample was machined using diamond turning (without oil) and was followed by an ultrapure water Megasonic rinse for 1 minute and a 1-minute alcohol rinse in a $40^{\circ} \mathrm{C}$ bath. A photograph of the Ag-plated copper sample after rf testing is shown in Fig. 48. The $\mathrm{TE}_{01}$ pulsed heating ring after test was strongly pronounced due to the severity of surface damage on the silver plating. A characteristic SEM image of the surface taken before the test is shown in Fig. 49(a) and an SEM image taken at the outer rim of the pulsed heating ring is shown in Fig. 49(b). This is the low temperature region within the pulsed heating ring and most of the damage at this location was due to cracking. Figure 50 shows a typical area of the plated surface in the center of the pulsed heating ring where the peak pulsed heating temperature $\left(T_{\max }\right)$ occurs. The surface damage and cracking density along with spalling of the plating was observed to be much higher at this location.

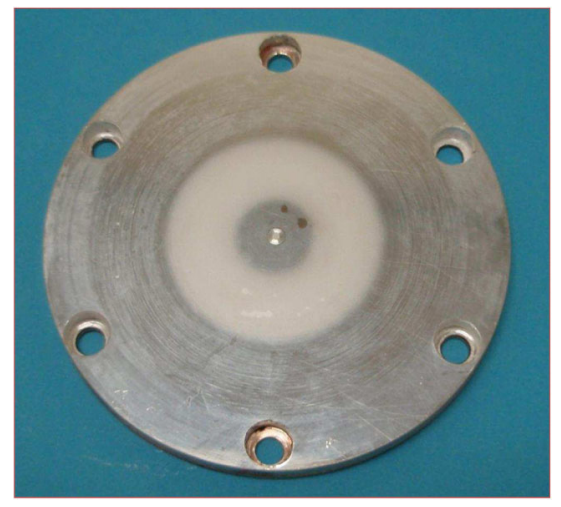

FIG. 48. Silver plated copper pulsed heating sample after rf testing.

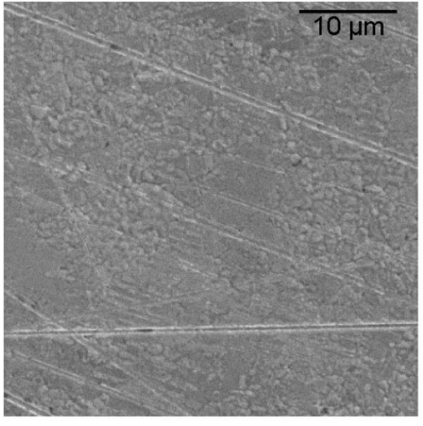

(a)

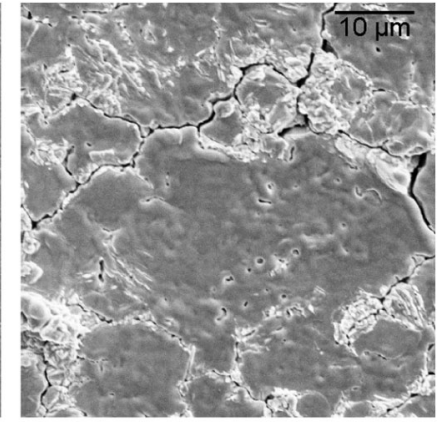

(b)
FIG. 49. Silver plated copper pulsed heating sample (a) before test and (b) after $110^{\circ} \mathrm{C}$ test (SEM image is taken at the outer edge of the pulsed heating ring). 


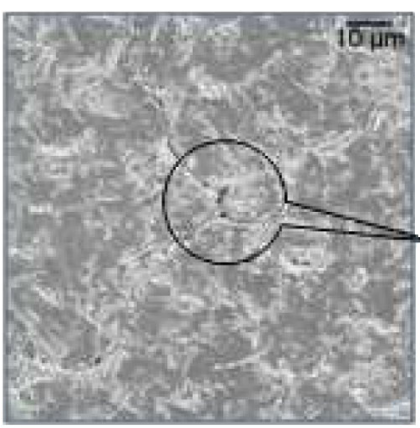

(a)

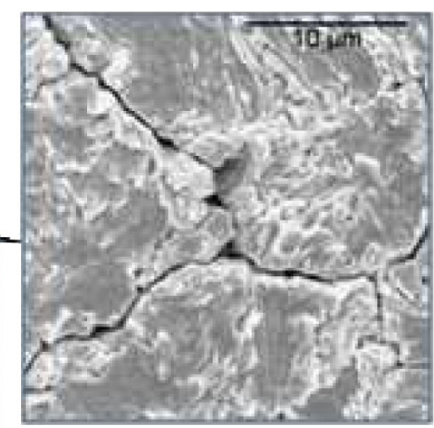

(b)
FIG. 50. SEM image taken in the center of the pulsed heating ring showing severe damage and cracking on the silver plating after the rf test.

\section{Glidcop ${ }^{\circledR}$}

In this study it has been shown that the harder materials significantly outperformed the softer materials and as a result Glidcop ${ }^{\circledR} \mathrm{C} 15715 \mathrm{Al}-15$ grade was selected as the next material to test. Glidcop ${ }^{\circledR}$ AL-15 is a low alumina content $(0.3 \mathrm{wt} \%)$ grade of dispersion strengthened copper. The copper matrix contains finely dispersed submicroscopic particles of $\mathrm{Al}_{2} \mathrm{O}_{3}$. The addition of aluminum oxide retards the recrystallization of the copper and increases the copper's resistance to thermal softening. The sample was machined from a clad extruded sheet and the surface was machined using diamond fly cutting and then cleaned with no chemical etch prior to the test. A photograph of the Glidcop ${ }^{\circledR}$ sample is shown in Fig. 51. The sample was received at SLAC with numerous scratch marks. The cause of these scratch marks is unclear. While it is not ideal nor desirable to have scratches on the surface, they did not seem to have an impact on the experimental results. The Glidcop ${ }^{\circledR}$ sample had the highest measured hardness value of all the samples tested in this study. There were two pulsed heating hot spots visible after rf testing the sample.

Additional surface damage on the sample was observed by SEM but was different than the other test samples.

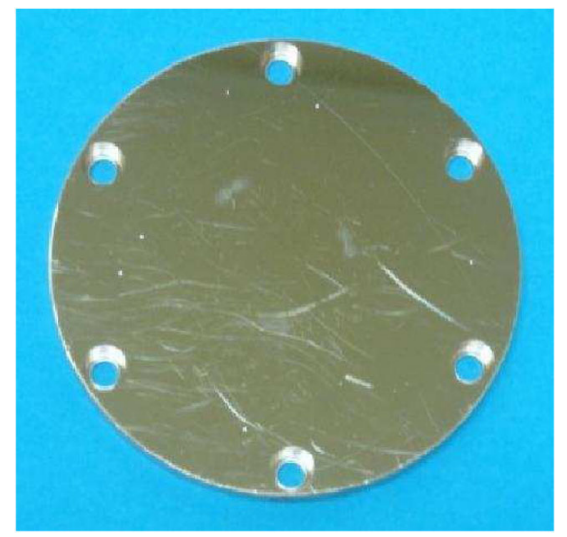

FIG. 51. Glidcop ${ }^{\circledR}$ pulsed heating sample after the $110^{\circ} \mathrm{C}$ test. The scratches on the surface occurred prior to testing the sample.

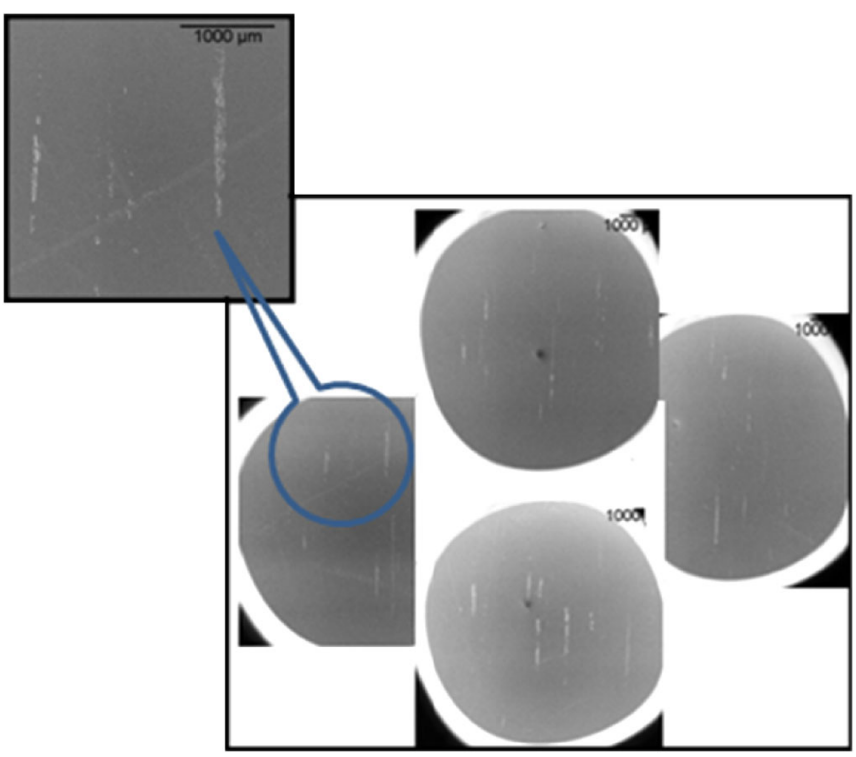

FIG. 52. Pulsed heating damage following straight lines and oriented in the same direction at various locations around the pulsed heating ring.

Small striation lines of pulsed heating damage were observed around the pulsed heating ring that had the same orientation irrespective of location. In the images shown in Fig. 52, these lines are vertical and can be seen at various locations around the pulsed heating ring. The cause is unclear but it could be due to material defects, or possibly surface imperfections created in the diamond fly-cutting process. Diamond fly cutting is different than a standard lathe where the cutter is fixed and the part being machined rotates on a spindle. In the diamond fly-cutting case the part is fixed and the cutter moves in a raster fashion which may have created surface defects that were preferentially impacted by pulsed heating. However, the diamond flycutting process was also used on some of the copper and copper zirconium samples tested in this study and the striation pattern was not observed in any other experiment.

A high magnification SEM image looking inside one of these narrow strips is shown in Fig. 53. The damage
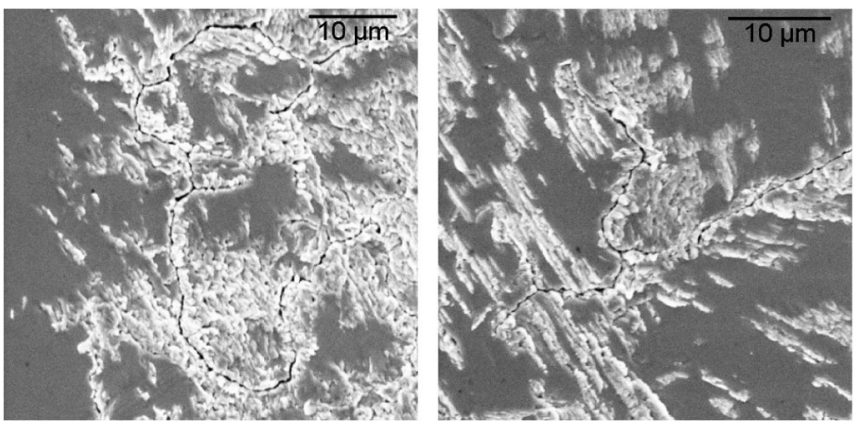

FIG. 53. Intergranular and transgranular surface fatigue extrusions observed within the Glidcop ${ }^{\circledR}$ pulsed heating striation lines. 

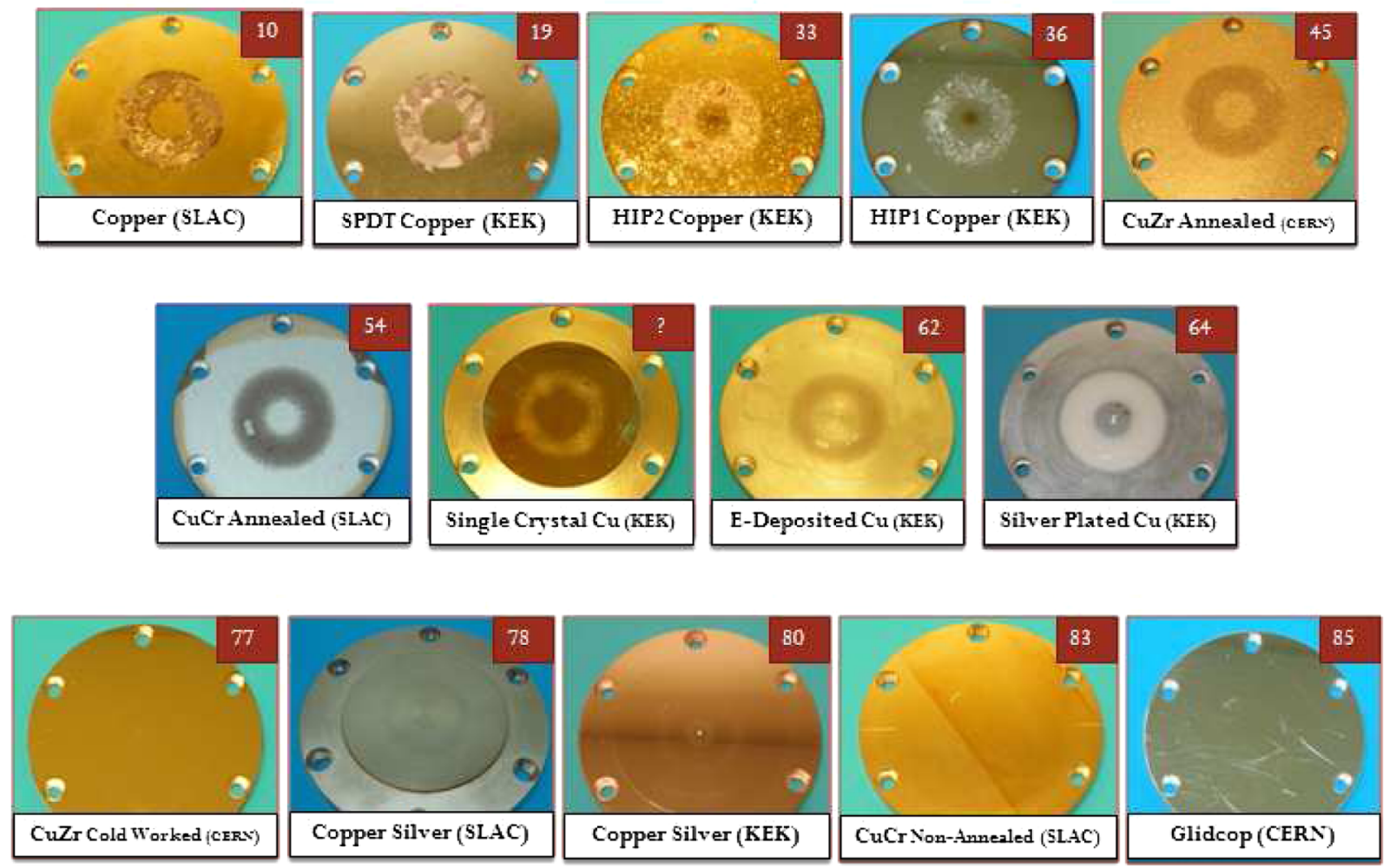

FIG. 54. Pulsed heating samples that have been tested to $110^{\circ} \mathrm{C}$. They are listed in order of material hardness values with the softest materials in the top row and the harder materials in the bottom row (hardness values are located in the top right corner of each photograph).

observed in these narrow strips shows surface extrusions and is consistent with what has been found on the other test samples discussed in this paper. Except for these small striations, the surface damage on the Glidcop ${ }^{\circledR}$ sample was minimal.

\section{CONCLUSIONS}

A series of pulsed heating experiments were conducted at SLAC National Accelerator Laboratory in collaboration with CERN and KEK. The motivation for this work is to better understand surface fatigue and the impact of highpower pulsed magnetic fields on copper and copper alloys. The samples tested were exposed to different machining and heat treatment processes and the materials used in this study included OFE copper, copper zirconium, copper chromium, HIP copper, single crystal copper, electroplated copper, Glidcop ${ }^{\circledR}$, copper silver, and silver plated copper. The test samples are shown in Fig. 54. Each of the samples was tested to within $10 \%$ of a peak pulsed heating temperature of $110^{\circ} \mathrm{C}$ for approximately $1 \times 10^{7} \mathrm{rf}$ pulses. The pulsed heating temperature was slightly higher for the silver plated copper sample due to its higher thermal conductivity. From left to right the samples are ordered according to their hardness value as measured by a Rockwell superficial hardness tester. The hardness values are shown in the top right corner of each photograph and the measurement units are HR15T. The surface damage due to pulsed heating was significantly more pronounced on the softer materials that are shown on the top row of Fig. 54. In contrast, the samples with hardness values $>70$ HR15T (bottom row) were minimally impacted by cyclic thermal fatigue. The impact of pulsed heating in this experiment was dominated by material hardness because material properties such as conductivity are weak functions of pulsed heating temperature. Likewise, all other physical properties of the materials tested were small compared to the variations in material hardness. Technologies for building accelerator structures made from the harder copper alloys are not yet completely developed but some testing in single cell structures has been conducted that shows some improvement over pure copper (see Refs. [15,16] for further details).

The material properties of the samples tested are shown in Table I. These values were utilized in calculating the maximum pulsed heating temperature that each of the different materials was subjected to in this study. The width of the pulsed heating ring was measured on the samples in order to determine the minimum pulsed heating temperature required to initiate surface damage. This data 
TABLE I. Physical properties for the materials tested in this study and the pulsed heating ring width and minimum temperature where the onset of pulsed heating damage occurs.

\begin{tabular}{|c|c|c|c|c|c|c|c|}
\hline Material & $\begin{array}{c}\text { Heat } \\
\text { capacity } \\
(\mathrm{J} / \mathrm{kg} / \mathrm{K})\end{array}$ & $\begin{array}{c}\text { Thermal } \\
\text { conductivity } \\
(\mathrm{W} / \mathrm{m} / \mathrm{K})\end{array}$ & $\begin{array}{c}\text { Electrical } \\
\text { conductivity } \\
\% \text { IACS }\end{array}$ & $\begin{array}{c}\text { Density } \\
(\mathrm{gm})\end{array}$ & $\begin{array}{c}\text { Maximum pulsed } \\
\text { heating temperature } \\
(\text { Ho }=600 \mathrm{kA} / \mathrm{m}) \\
(\text { Celsius })\end{array}$ & $\begin{array}{l}\text { Pulsed heating } \\
\text { ring width } \\
(\mathrm{mm})\end{array}$ & $\begin{array}{c}\text { Onset of pulsed } \\
\text { heating damage } \\
\text { (Celsius) }\end{array}$ \\
\hline Copper (SLAC) & 394 & 391 & $101^{\mathrm{a}}$ & 8.94 & 110 & 10.7 & 66 \\
\hline SPDT $\mathrm{Cu}$ & 394 & 391 & $(100)^{\mathrm{b}}$ & 8.94 & 110 & 4.7 & 100 \\
\hline $\mathrm{HIP} 2 \mathrm{Cu}$ & 394 & 391 & $(100)^{\mathrm{b}}$ & 8.94 & 110 & 11.9 & 57 \\
\hline CuZr (annealed) & 394 & 367 & $78-82^{a}$ & 8.89 & 114 & 12.1 & 58 \\
\hline $\mathrm{CuCr}$ (annealed) & 385 & 324 & $83.5^{\mathrm{a}}$ & 8.89 & 121 & 13.4 & 52 \\
\hline Single crystal $\mathrm{Cu}$ & 394 & 391 & $(100)^{\mathrm{b}}$ & 8.94 & 110 & 5.4 & 97 \\
\hline E-deposited $\mathrm{Cu}$ & 394 & 391 & $(100)^{\mathrm{b}}$ & 8.94 & 110 & 8.5 & 80 \\
\hline Silver plated $\mathrm{Cu}$ & 233 & 429 & $(105)^{\mathrm{b}}$ & 10.5 & 125 & 12.5 & 60 \\
\hline CuZr (cold worked) & 394 & 367 & $82-83$ & 8.89 & 114 & & $>114$ \\
\hline $\mathrm{CuAg}$ (SLAC) & 385 & 346 & $97-100.1$ & 8.89 & 117 & 6.2 & 100 \\
\hline CuAg (KEK) & 385 & 346 & 97 & 8.89 & 117 & 4.7 & 107 \\
\hline $\mathrm{CuCr}$ (nonannealed) & 385 & 324 & 83.5 & 8.89 & 121 & & $>121$ \\
\hline Glidcop & 384 & 335 & 91.5 & 8.91 & 119 & & $>119$ \\
\hline
\end{tabular}

${ }^{\text {a}}$ Precise information not available.

${ }^{\mathrm{b}}$ Measured values prior to heat treatment.

is shown in the last column of the table. All of the images shown in this paper are available as supplemental material which will enable higher resolution viewing (see Ref. [20]). In general, the level of pulsed heating surface damage was less on the small grain samples. This may be due to the small grain boundaries limiting the propagation of surface extrusions which were observed to terminate on grain boundaries.

Other notable observations in this study included: (i) Material extrusions from intergranular and transgranular fractures were observed to varying degrees on all the samples tested to $110^{\circ} \mathrm{C}$ except for the cold worked $\mathrm{CuZr}$ sample which was not noticeable until after the $150^{\circ} \mathrm{C}$ test run. (ii) At low cyclic temperatures, grain boundaries were observed to be the initial locations impacted by pulsed heating. (iii) At high cyclic temperatures $\left(110^{\circ} \mathrm{C}\right)$, metallography revealed subsurface intergranular damage that extended $20-40 \mu \mathrm{m}$ below the surface on heat treated copper. (iv) Two different types of surface damage were observed on grain surfaces. Transgranular surface extrusions oriented in different directions and terminating on grain boundaries created a weave pattern and the second type of damage was due to extrusions through micropits. It is speculated that the differences between these two may be due to a difference in crystallographic orientation. (v) A dependence of the severity of degradation of the surface on crystallographic orientation was determined using electron backscattered diffraction technology. (vi) Within the $\mathrm{TE}_{01}$ pulsed heating ring of single crystal copper, the material hardness increased almost proportionally to the pulsed heating temperature. A radial distribution of hardness and cyclic temperature rise showed that a threshold of approximately $60 \mathrm{~K}$ has to be overcome to initiate surface hardening. (vii) Nonheat treated samples which had a higher material hardness and smaller grain size significantly outperformed the heat treated samples.

\section{ACKNOWLEDGMENTS}

The authors would like to thank Scott Beebe, John Eichner, Andrew Haase, Erik Jongewaard, James Lewandowski, Dave Martin, Chris Pearson, Rich Talley, Bob Vandersyl, Dian Yeremian, and Chuck Yoneda for their help and support. This work is supported by Department of Energy Contract No. DE-AC0376 SF00515.

[1] P. B. Wilson, AIP Conf. Proc. 397, 191 (1997).

[2] I. H. Wilson, Technical Report No. CLIC-Note-52, CERN, Geneva, 1987.

[3] D. P. Pritzkau and R.H. Siemann, Phys. Rev. ST Accel. Beams 5, 112002 (2002).

[4] W. Wuensch, in Proceedings of the 11th European Particle Accelerator Conference, Genoa, 2008 (EPS-AG, Genoa, Italy, 2008).

[5] S. Calatroni, H. Neupert, and M. Taborelli, CLIC Note Report No. 615, CERN 2004.

[6] A. Grudiev and W. Wuensch, Technical Report No. CLICNote-773, CERN 2008.

[7] R. Jones, C. Adolphsen, J. Wang, and L. Zenghai, Phys. Rev. ST Accel. Beams 9, 102001 (2006). 
[8] V. Dolgashev, S. Tantawi, Y. Higashi, and T. Higo, in Proceedings of the 11th European Particle Accelerator Conference, Genoa, 2008 (Ref. [4]), pp. 742-744.

[9] M. Yeddulla, S. Tantawi, J. Guo, and V. Dolgashev, IEEE Trans. Microwave Theory Tech. 57, 1516 (2009).

[10] ANSYS, www.ansoft.com/products/hf/hfss/ (2010).

[11] S. Tantawi, Phys. Rev. ST Accel. Beams 8, 042002 (2005).

[12] D. Kajfez, IEEE Trans. Microwave Theory Tech. 51, 512 (2003).

[13] G. Caryotakis, in Proceedings of the Particle Accelerator Conference, Vancouver, BC, Canada, 1997 (IEEE, New York, 1997).

[14] D. P. Pritzkau, Ph.D. thesis, Stanford Linear Accelerator Center, 2001; see Chap. 3, Eq. 3.32.

[15] V. Dolgashev, S. Tantawi, Y. Higashi, and B. Spataro, Appl. Phys. Lett. 97, 171501 (2010).
[16] S. Tantawi, V. Dolgashev, Y. Higashi, and B. Spataro, AIP Conf. Proc. 1299, 29 (2010), http://link.aip.org/link/? APC/1299/29/1.

[17] V. Dolgashev, in Proceedings of the 20th Particle Accelerator Conference, Portland, OR, 2003 (IEEE, New York, 2003), Vol. 2, pp. 1267-1269.

[18] V. A. Dolgashev and S. G. Tantawi, in Proceedings of the 8th European Particle Accelerator Conference, Paris, 2002 (EPS-IGA and CERN, Geneva, 2002), pp. 2139-2141.

[19] M. Aicheler, S. Sgobba, G. Arnau-Izquierdo, M. Taborelli, S. Calatroni, H. Neupert, and W. Wuensch, Int. J. Fatigue 33, 396 (2011).

[20] See supplemental material at http://link.aps.org/supplemental/10.1103/PhysRevSTAB.14.041001 for larger and higher resolution images of the figures contained in this paper. 